\title{
Non-Linear Response of a Fluid Valve
}

by

Hichem Bouguerra

Thesis submitted to the Faculty of the

Virginia Polytechnic Institute and State University

in partial fulfillment of the requirements for the degree of

Master of Science

in

Engineering Mechanics

APPROVED:

Ali H. Nayfeh, Chairmah

Dean T. Mook

S. L. Hendricks

August 1987

Blacksburg. Virginia 


\section{Non-Linear Response of a Fluid Valve}

by

Hichem Bouguerra

Ali H. Nayfeh, Chairman

Engineering Mechanics

(ABSTRACT)

The method of multiple scales is used to study the nonlinear response of a relief valve under a constant static pressure and a sinusoidal dynamic pressure that can be generated by variations in the pneumatic transmission lines. Under certain conditions, large vibrations may occur, causing unsatisfactory performance as well as undesirable noise. In this theoretical analysis, the valve is modeled by a continuous spring fixed at one end and attached to a valve ball at the other end. The ball rests on a valve seat having nonlinear spring characteristics. The method of multiple scales is used to determine the non-linear response of the valve for the cases of harmonic, higher-harmonic, subharmonic and combination resonances. Conditions are determined for the onset and stability of large-amplitude oscillations. 


\section{Acknowledgements}

I owe many thanks to my advisor, Professor A. H. Nayfeh, whose advice and assistance were invaluable throughout this study.

I would also like to thank Professors D. T. Mook and S. L. Hendricks for serving as members on my examining committee and for reviewing this thesis. 


\section{Table of Contents}

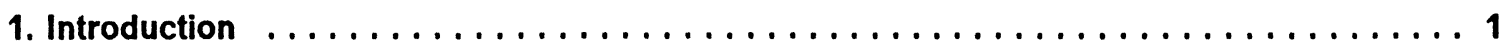

1.1 Problem Description $\ldots \ldots \ldots \ldots \ldots \ldots \ldots \ldots \ldots \ldots \ldots \ldots \ldots \ldots \ldots \ldots$

1.2 Literature Review $\ldots \ldots \ldots \ldots \ldots \ldots \ldots \ldots \ldots \ldots \ldots \ldots \ldots \ldots$

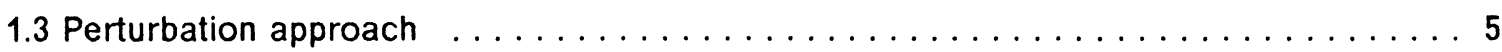

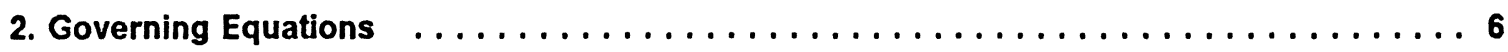

2.1 Lagrange's equations of motion $\ldots \ldots \ldots \ldots \ldots \ldots \ldots \ldots \ldots \ldots \ldots \ldots \ldots \ldots \ldots \ldots \ldots \ldots$

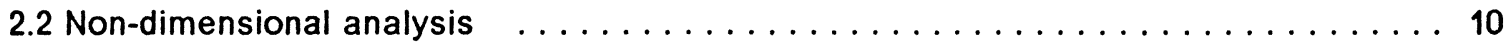

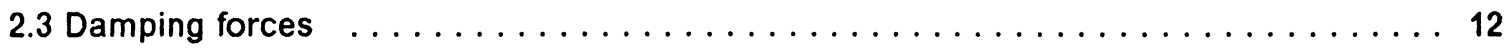

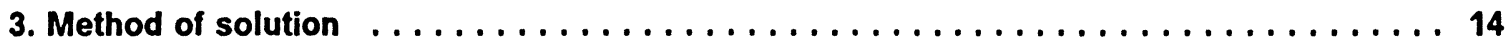

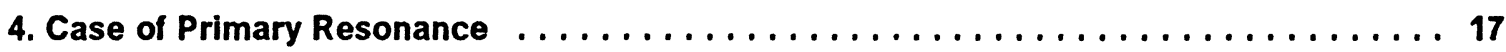

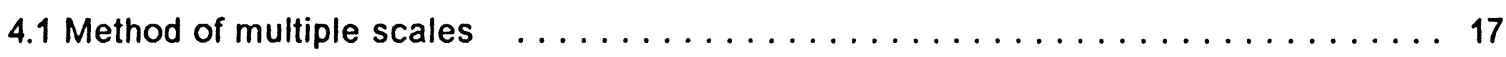

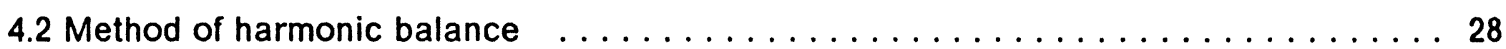

5. Case of Subharmonic resonance of order $1 / 3 \ldots \ldots \ldots \ldots \ldots \ldots \ldots$ 
6. Case of Superharmonic resonance of order 3

7. Case of Subharmonic Resonance of Order One-Half 44

8. Case of Combination Resonance 53

9. Case of Superharmonic resonance of order 2 59

10. Summary and Conclusions

References 64

Appendix A. Solvability Conditions 66

Appendix B. Method of harmonic balance 68

Vita 74 


\section{List of Illustrations}

Figure 1. Experimental relief valve $\ldots \ldots \ldots \ldots \ldots \ldots \ldots \ldots \ldots \ldots$

Figure 2. Schematic model of a relief valve $\ldots \ldots \ldots \ldots \ldots \ldots$

Figure 3. A representative frequency-response curve for the case of primary

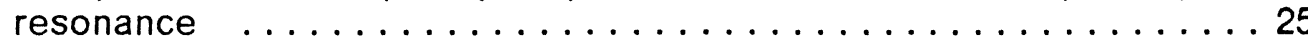

Figure 4. Representative variations of the response amplitude with the excitation amplitude for several detunings, primary resonance . . . . . . 26

Figure 5. Frequency-response curve for the case of subharmonic resonance of

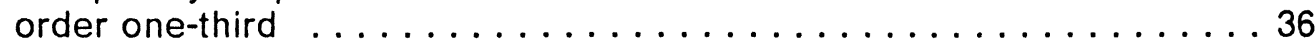

Figure 6. Response amplitude as a function of the excitation amplitude for dif-

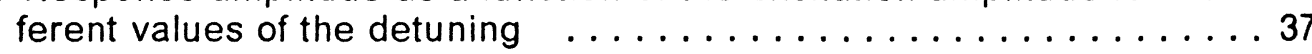

Figure 7. Comparison of frequency-response curves $\ldots \ldots \ldots \ldots \ldots \ldots$

Figure 8. Frequency-response curve for the case of subharmonic resonance of

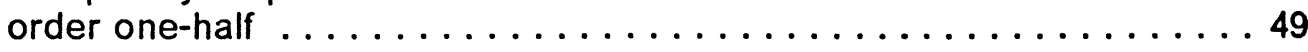

Figure 9. Response amplitude as a function of the excitation amplitude for the case of subharmonic resonance of order one-half $\ldots \ldots \ldots \ldots$

Figure 10. Response amplitude as a function of the excitation amplitude fol the case of subharmonic resonance of order one-half $\ldots \ldots \ldots \ldots \ldots$

Figure 11. Frequency-response curve for the case of combination resonance of

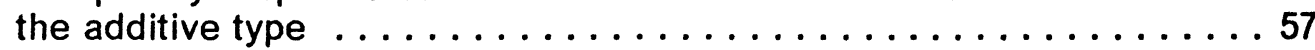

Figure 12. Effective spring constant due to second harmonic. $\ldots \ldots \ldots \ldots \ldots$ 


\section{Introduction}

\subsection{Problem Description}

The linear wave equation is one of the best known and well studied equations in mathematical physics. In its one dimensional form

$$
\frac{\partial^{2} u}{\partial t^{2}}=c^{2} \frac{\partial^{2} u}{\partial x^{2}}
$$

with $\mathrm{c}$ being constant, it governs the linear theories of sound waves in a pipe, longitudinal and transverse oscillations of an elastic string, electric signals in transmission lines, and in our study it represents the motion of a helical spring of a fluid valve.

Figure 1 shows a schematic repesentation of an experimental relief valve used to protect a fluid system from overpressure. In its simplest design the valve consists of a ball held by a helical spring against a valve seat having non-linear spring characterestics. The dynamic behavior of the whole system can be modeled by equation (1.1) subject to proper boundary conditions. In the case at hand, the helical 


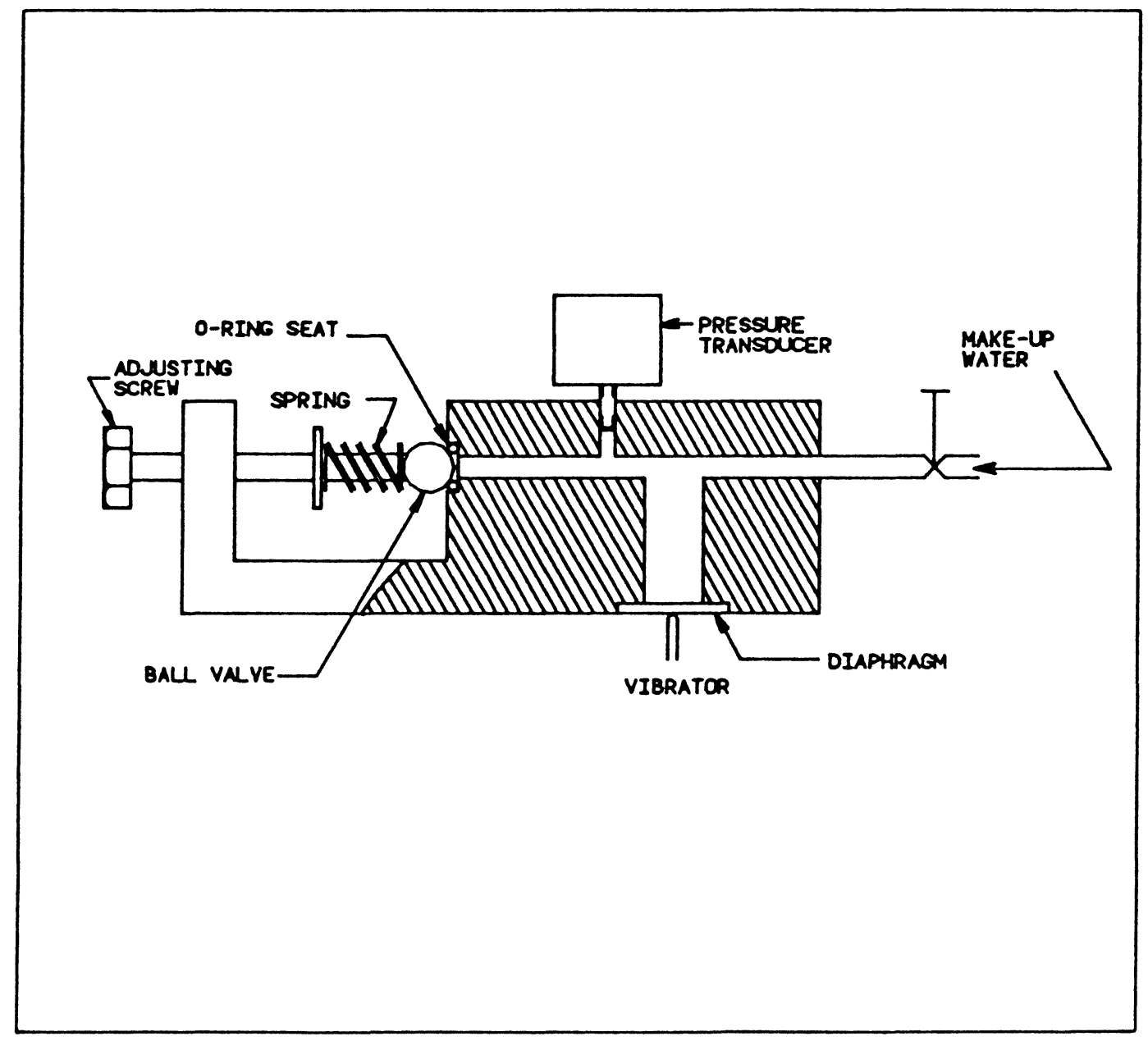

Figure 1. Experimental relief valve 
spring is considered as a distributed parameter system and its motion is governed by equation (1.1) with proper damping terms added. One end of the spring is taken to be fixed, whereas the other end is taken to be restrained by a force due to the valve seat and subject to a constant static pressure and a sinusoidally varing dynamic pressure resulting from variations in the pneumatic transmission lines.

\subsection{Literature Review}

Johnson and Wandling [6] showed that actual popping pressure of a relief valve under dynamic loading is frequency dependent. They showed that because of the spring dynamics the actual popping pressure may be a fraction of the static popping pressure. Both experimental and theoretical results, which appear to follow somewhat the same trend, confirm that the actual popping pressure does vary greatly from that for which the valve was statically set. Dokainish and Elmadany [3] used the method of harmonic balance to analyze the influence of the nonlinear spring characteristics in the absence of damping. They studied the cases of primary resonance, superharmonic resonance of order-three, and subharmonic resonance of order one-third. Their assumed solutions do not contain the second harmonic and hence their results are valid only for static pressures that are the order of the dynamic component. Paslay and Gurtin [14] analysed primary and third harmonic resonances in the response of a linear system resting on a non-linear spring to a harmonic excitation. Nayfeh and Asfar [12] used the method of multiple scales $[10,11]$ to analyse the response and the stability of a bar constrained by a non-linear spring to a harmonic 
excitation for the cases of primary, subharmonic, superharmonic, combination and ultrasubharmonic resonances. Collins [2] used a perturbation procedure to obtain solutions for systems governed by non-linear wave equations. He showed that, even though solutions of linear equations under forced oscillations become infinite at or near resonant frequencies, bounded solutions to certain non-linear wave equations appear to exist at all frequencies. Albowitz and his associates [1] outlined a method for obtaining periodic solutions to systems governed by non-linear wave equations. They used regular perturbation theory but rescaled variables when the theory breaks down, i.e. at or near resonance. Nakashima and his associates [9] studied the effects of configuration of the pressure lines and elements of the regulator, such as the mass of the valve spring system, spring constant etc. on the response of the regulator. In their theoretical analysis, the motion of the spring was modeled as a damped vibration of one-degree-of-freedom system under external forces. The results were then compared with those obtained experimentally in order to clarify their validity and determine the conditions under which occasional vibrations may arise during operation. They concluded that occasional vibrations of the regulator may arise when the resistance of the pipe line becomes large. They also observed that when the adjusting pressure decreases below a standard value, these vibrations may be easily generated due to severe pressure changes in the chambers of the regulator in the transient response process. Hayashi and his associates [4] studied the stability of a system consisting of a nozzle-flapper valve and a pipe line. They concluded that the most effective nonlinear characteristic for limiting the amplitude of the unstable oscillations is the collision of the flapper with the nozzle tip. To represent the flapper restoring force, they proposed a nonlinear spring force. Basic equations of the system are then derived to analyze the character of self-sustained oscillations and their selective occurrence. 


\subsection{Perturbation approach}

In this investigation the method of multiple scales is used to analyse the response of the relief valve. First-order ordinary differential equations are derived for the evolution of the amplitudes and the phases with damping, quadratic and cubic nonlinearities, for the following resonances: primary resonance $\Omega \cong \omega_{n}$; subharmonic resonance of order one-third $\Omega \cong 3 \omega_{n}$; superharmonic resonance of order three $\Omega \cong \frac{1}{3} \omega_{n}$; subharmonic resonance of order one-half $\Omega \cong 2 \omega_{n}$; superharmonic resonace of order two $\Omega \cong \frac{1}{2} \omega_{n}$; and combination resonance of the form $\Omega \cong \omega_{n} \overline{+} \omega_{m}$, where the $\omega_{\mathrm{s}}$ are the linear undamped natural frequencies of the system. The evolution equations are used to determine the steady-state responses and their stability. 


\section{Governing Equations}

\subsection{Lagrange's equations of motion}

In many applications the usual assumption of a massless spring, which leads to a classical second-order system, is inadequate. The effect of mass-spring has been studied and shown to cause what is called "surging" [16].

This investigation considers a relief valve with a helical spring having a finite mass. The spring is represented mathematically as a distributed parameter model. The well known extended Hamilton principle [4] is then applied to derive the equations of motion. A schematic representation of the relief valve is shown in figure 2.

The kinetic energy density of the system modeled by figure 2 is of the form

$$
\hat{T}=\frac{1}{2} \frac{M}{L}[\dot{u}(x, t)]^{2}+\frac{1}{2} m[\dot{u}(L, t)]^{2}
$$

The potential energy density can be written as 


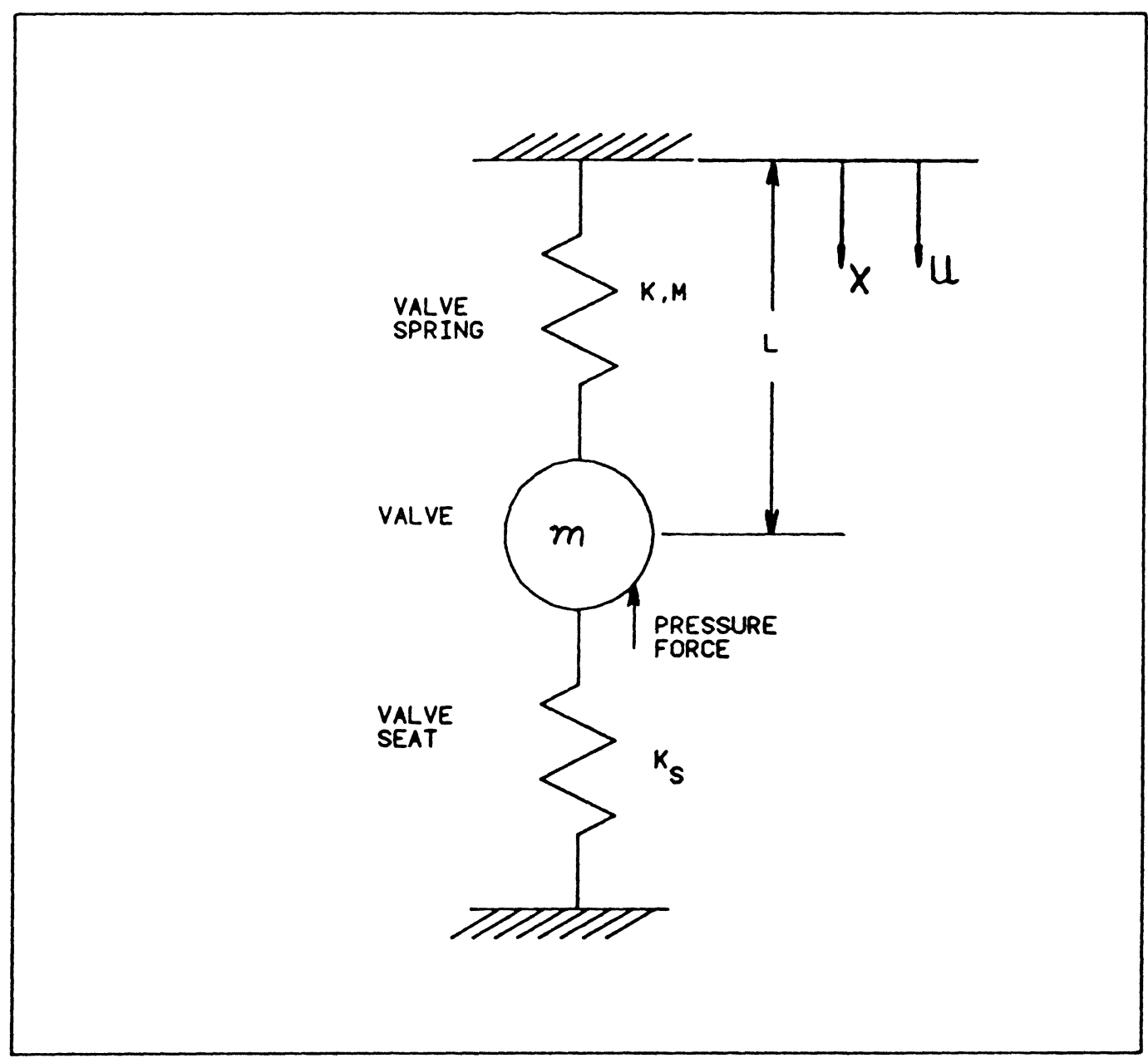

Figure 2. Schematic model of a relief valve 


$$
\hat{V}=\frac{1}{2} K L u^{\prime 2}(x, t)+\frac{1}{2} F_{s} u(L, t)
$$

where $F$, is the force generated by the valve seat and is defined according to

$$
F_{s}=\alpha u+\beta u^{3} I_{x=L}
$$

The non-conservative virtual work density is defined according to

$$
\hat{W}_{\mathrm{nc}}=\left(\Phi_{0}+\Phi_{1} \cos \Omega \mathrm{t}\right) \mathrm{u}(\mathrm{L}, \mathrm{t})
$$

The dots denote partial derivatives with respect to the time $t$ and the primes denote partial derivatives with respect to the space coordinate $\mathrm{x}$, and the subscript "nc" refers to non-conservative.

The equations of motion are obtained by means of the extended Hamilton principle, which can be expressed as

$$
\int_{t_{1}}^{t_{2}}\left[\int_{0}^{L} \delta \hat{L} d x+\delta \hat{W}_{n c}+\delta L_{0}\right] d t=0 \quad\left\{\begin{array}{l}
\delta u=0, \quad 0 \leq x \leq L \\
\text { at } t=t_{1}, t_{2}
\end{array}\right.
$$

where $\delta u$ is an arbitrary virtual displacement taken to be zero at times $t_{1}$ and $t_{2}$ along the length of the spring, $\hat{L}$ is the Lagrangian density having the form

$$
\hat{L}=\frac{1}{2} \frac{M}{L} \dot{u}^{2}-\frac{1}{2} K L u^{\prime 2}
$$

and $L_{0}$ is the discrete component of the Lagrangian which can be written as 


$$
L_{0}=\frac{1}{2} m \dot{u}^{2}(L, t)-\left.\frac{1}{2}\left(\alpha u^{2}+\beta u^{4}\right)\right|_{x=L}
$$

The Lagrangian density variation can be written as

$$
\delta \hat{L}=\frac{\partial \hat{L}}{\partial \dot{u}} \delta \dot{u}+\frac{\partial \hat{L}}{\partial u^{\prime}} \delta u^{\prime}
$$

Similarly variations in $L_{0}$ and $\hat{W}_{n c}$ are computed and substituted along with (2.7) into (2.4) to obtain the Lagrange's differential equation of motion as

$$
\frac{\partial^{2} u}{\partial t^{2}}=\frac{K L^{2}}{M} \frac{\partial^{2} u}{\partial x^{2}}
$$

subject to the boundary conditions

$$
\begin{array}{cc}
u=0 & \text { at } x=0 \\
m \frac{\partial^{2} u}{\partial t^{2}}+K L \frac{\partial u}{\partial x}+\alpha u+\beta u^{3}=\Phi_{0}+\Phi_{1} \cos \Omega t & \text { at } x=L
\end{array}
$$

Note that the arbitrariness of the virtual displacement was taken into account in the process of passing through the extended Hamilton principle to the equations of motion. Of course, the virtual displacement $\delta u$ is arbitrary and can be assigned values at will provided compatibilty with the system constraints, such as geometric boundary conditions, is satisfied.

For the problem at hand, the geometric boundary conditions dictate that $u=0$ at the end $x=0$, whereas at the end $x=L$, the restoring force due to the valve seat, the 
force in the valve spring and the excitation forces must obey Newton's second law when applied to the valve mass $\mathrm{m}$.

\subsection{Non-dimensional analysis}

Non-dimensionalization of the differential equations of motion (2.8)-(2.10) can be achieved by introducing the following non-dimensional variables:

$$
u^{*}=\frac{u}{U}, \quad t^{*}=\omega_{0} t, \quad x^{*}=\frac{x}{L}
$$

where $\omega_{0}=\sqrt{\frac{K}{M}}$ and $U$ is a representative longitudinal displacement introduced for convenience. The new constant $\omega_{0}$ was introduced arbitrarily and was chosen to be the fundamental natural frequency of the valve spring for convenience only.

By means of the chain rule, the time derivatives can be expressed as

$$
\begin{aligned}
& \frac{\partial}{\partial t}=\omega_{0} \frac{\partial}{\partial t^{*}} \\
& \frac{\partial^{2}}{\partial t^{2}}=\omega_{0}^{2} \frac{\partial^{2}}{\partial t^{2}}
\end{aligned}
$$

Similarly the space derivatives can be expressed as 


$$
\begin{aligned}
& \frac{\partial}{\partial x}=\frac{1}{L} \frac{\partial}{\partial x^{*}} \\
& \frac{\partial^{2}}{\partial x^{2}}=\frac{1}{L^{2}} \frac{\partial^{2}}{\partial x^{*}}
\end{aligned}
$$

Substituting (2.11)-(2.15) into (2.8)-(2.10), one obtains the differential equations of motion in non-dimensional form as

$$
\begin{array}{cl}
u_{t t}=u_{x x} & \\
u=0 & \text { at } x=0 \\
u_{t t}+\alpha_{1} u_{x}+\alpha_{2} u+\alpha_{3} u^{3}=F_{0}+F_{1} \cos \Omega t & \text { at } x=1
\end{array}
$$

where

$$
\begin{aligned}
& \alpha_{1}=\frac{M}{m} \\
& \alpha_{2}=\frac{\alpha M}{m K} \\
& \alpha_{3}=\frac{\beta M U^{2}}{m K} \\
& F_{0}=\frac{\Phi_{0} M}{m U K} \\
& F_{1}=\frac{\Phi_{1} M}{m U K}
\end{aligned}
$$


Note that the asterisks on $\mathrm{u}, \mathrm{x}$ and $\mathrm{t}$, denoting non-dimensionality of the variables, have been dropped for ease of notation. Also, notations of partial derivatives of $u$ with respect to $t$ and $x$ have been changed to $u_{t}$ and $u_{x}$, respectively.

\subsection{Damping forces}

The system shown in figure 1 is subjected to many damping forces due to various causes that may include

1. Internal hesteresis in the spring material.

2. Air resistance.

3. Damping due to friction in the end turns.

4. Damping due to loss of energy in the supports.

It would be hopelessly complicated if one tries to mathematically model each one of these damping sources. However, in many applications and with a reasonable accuracy, the damping force acting on the system can be assumed proportional to the velocity of the motion. Thus, introducing a damping coefficient $\mu_{1}$ as the constant of proportionality, one can add the damping force to the equation of motion (2.16) to obtain

$$
u_{x x}=u_{t t}+2 \mu_{1} u_{t}
$$


The boundary conditions are

$$
\begin{array}{cc}
u=0 & \text { at } x=0 \\
u_{\mathrm{tt}}+\alpha_{1} u_{\mathrm{x}}+\alpha_{2} u+\alpha_{3} u^{3}=F_{0}+F_{1} \cos \Omega t & \text { at } x=1
\end{array}
$$

The coefficient 2 in (2.24) is introduced for reasons that will be obvious later when complex quantities are introduced. 


\section{Method of solution}

The method of multiple scales $[1,2]$ is used to detemine a uniform first-order expansion of the solution of equations (2.16)-(2.18). To this end, we express the solution as the sum of a static component and a dynamic component; that is,

$$
u(x, t)=b x+v(x, t)
$$

where b satisfies

$$
\left(\alpha_{1}+\alpha_{2}\right) b+\alpha_{3} b^{3}=F_{0}
$$

and $v$ satisfies

$$
\begin{gathered}
v_{\mathrm{xx}}=v_{\mathrm{tt}}+2 \mu_{1} v_{\mathrm{t}} \\
v=0 \quad \text { at } x=0 \\
v_{\mathrm{tt}}+\alpha_{1} v_{\mathrm{x}}+\left(\alpha_{2}+3 \alpha_{3} b^{2}\right) v+3 \alpha_{3} b v^{2}+\alpha_{3} v^{3}=F_{1} \cos \Omega t \quad \text { at } x=1
\end{gathered}
$$

We let 


$$
v=\varepsilon^{\lambda} w
$$

in (3.3)-(3.5) and obtain

$$
\begin{gathered}
w_{x x}=w_{t t}+2 \mu_{1} w_{t} \\
w=0 \quad \text { at } x=0 \\
w_{t t}+\alpha_{1} w_{x}+\alpha_{4} w+\varepsilon^{\lambda} \alpha_{5} w^{2}+\varepsilon^{2 \lambda} \alpha_{3} w^{3}=\frac{F_{1}}{\varepsilon} \cos \Omega t \quad \text { at } x=1
\end{gathered}
$$

where

$$
\begin{gathered}
\alpha_{4}=\alpha_{2}+3 \alpha_{3} b^{2} \\
\alpha_{5}=3 \alpha_{3} b
\end{gathered}
$$

The parameter $\varepsilon$ is a small but finite non-dimensional quantity that is introduced as a book-keeping device.

To determine a second-order approximation to the solution of (3.7)-(3.9), we assume an expansion of the form

$$
w(x, t ; \varepsilon)=w_{0}\left(x, T_{0}, T_{1}, T_{2}\right)+\varepsilon w_{1}\left(x, T_{0}, T_{1}, T_{2}\right)+\varepsilon^{2} w_{2}\left(x, T_{0}, T_{1}, T_{2}\right)+\cdots
$$

where $T_{0}=t$ is a fast scale, characterizing motions at the natural frequencies and the excitation frequency $\Omega$, and $T_{1}=\varepsilon t$ and $T_{2}=\varepsilon^{2}$ are slow scales, characterizing modulations of the amplitudes and phases due to damping, non-linearity and any resonances. Thus the time derivatives transform according to 


$$
\frac{\partial}{\partial t}=D_{0}+\varepsilon D_{1}+\varepsilon^{2} D_{2}+\cdots
$$

and

$$
\frac{\partial}{\partial t^{2}}=D_{0}^{2}+2 \varepsilon D_{0} D_{1}+\varepsilon^{2}\left(D_{1}^{2}+2 D_{0} D_{2}\right)+\cdots
$$

where

$$
D_{n}=\frac{\partial}{\partial T_{n}}
$$

Substituting (3.12)-(3.14) into (3.7)-(3.9) and equating coefficients of like powers of $\varepsilon$ on both sides of each equation, one obtains separate systems of linear partialdifferential equations that can be solved in succession. The system of equations obtained at the highest level of approximation needs not be solved. A great deal of information about the solution can be obtained by simply applying the solvability conditions to this system of equations as described in Appendix A. A tremendous reduction in the algebra involved can be achieved if the solution of each system at each level of approximation is expressed in complex form.

The method of multiple scales, though a little more involved, proves to be superior in many applications to other methods such as the Lindstedt-Poincare method, the method of harmonic balance and the method of averaging. This fact is best shown when treating damped systems and as in our case a system that has quadratic and cubic non-linearities along with damping. For more detailed explanation of this method, the reader is referred to the textbooks by Nayfeh [1] and Nayfeh and Mook [2]. 


\section{Case of Primary Resonance}

\subsection{Method of multiple scales}

This resonance is excited whenever $\boldsymbol{\Omega}$, the excitation frequency, is near any of the linear natural frequencies $\omega_{n}$. Assuming that no other resonances exist, for instance a combination resonance could occur simultaneously with a primary resonance, one introduces a detuning parameter $\sigma$ to quantitatively relate the natural frequency $\omega_{n}$ to the excitation frequency $\Omega$ according to

$$
\boldsymbol{\Omega}=\omega_{n}+\varepsilon^{2} \sigma
$$

To analyze this case, one must first reorder the damping, the excitation and the nonlinearity so that they appear in the same solvability condition. To this end, we let

$$
\lambda=1 \quad, \quad \mu_{1}=\varepsilon^{2} \mu
$$




$$
\frac{F_{1}}{\varepsilon^{\lambda}}=\varepsilon^{2} f_{1}
$$

substitute (4.1)-(4.3) into (3.7)-(3.9), and obtain

$$
\begin{gathered}
w_{x x}=w_{t t}+2 \varepsilon^{2} \mu w_{t} \\
w=0 \quad \text { at } x=0 \\
w_{t t}+\alpha_{1} w_{x}+\alpha_{4} w+\varepsilon \alpha_{5} w^{2}+\varepsilon^{2} \alpha_{3} w^{3}=\varepsilon^{2} f_{1} \cos \Omega t \quad \text { at } x=1
\end{gathered}
$$

Substituting (3.12)-(3.14) into (4.4)-(4.6) and equating coefficients of like power of $\varepsilon$ on both sides of each equation yields

Order $\varepsilon^{0}:$

$$
\begin{array}{ll}
w_{0}=0 & \text { at } x=0 \\
D_{0 x x}^{2} w_{0}+\alpha_{1} w_{0 x}+\alpha_{0}^{2} w_{0}=0 & \text { at } x=0
\end{array}
$$

Order $\varepsilon$ :

$$
\begin{array}{cc}
w_{1 x x}=D_{0}^{2} w_{1}+2 D_{0} D_{1} w_{0} & \\
w_{1}=0 & \text { at } x=0 \\
D_{0}^{2} w_{1}+\alpha_{1} w_{1 x}+\alpha_{4} w_{1}=-2 D_{0} D_{1} w_{0}-\alpha_{5} w_{0}^{2} & \text { at } x=1
\end{array}
$$


Order $\varepsilon^{2}$ :

$$
\begin{array}{cc}
w_{2 x x}= & D_{0}^{2} w_{2}+2 D_{0} D_{1} w_{1}+\left(2 D_{0} D_{2}+D_{1}^{2}\right) w_{0}+2 \mu D_{0} w_{0} \\
w_{2}=0 & \text { at } x=0 \\
D_{0}^{2} w_{2}+\alpha_{1} w_{2 x}+\alpha_{4} w_{2}=-2 D_{0} D_{1} w_{1}-\left(2 D_{0} D_{2}+D_{1}^{2}\right) w_{0}-2 \alpha_{5} w_{0} w_{1} \\
-\alpha_{3} w_{0}^{3}+f_{1} \cos \Omega T_{0} \quad \text { at } x=1
\end{array}
$$

The solution of equations (4.7)-(4.9) can be expressed as

$$
w_{0}=\sum_{s=1}^{\infty} A_{s}\left(T_{1}, T_{2}\right) \frac{\sin \omega_{s} x}{\sin \omega_{s}} e^{i \omega_{s} T_{0}}+c c
$$

where cc stands for the complex conjugate of the preceding terms and the $\omega_{\mathbf{q}}$ are the linear undamped natural frequencies of the system given by the following characteristic equation:

$$
\alpha_{1} \omega_{s}+\left(\alpha_{4}-\omega_{s}^{2}\right) \tan \omega_{s}=0
$$

Up to this level of aproximation, the $A_{2}$ are unknown complex functions of the slow scales $T_{1}$ and $T_{2}$. They are determined by imposing the solvability conditions at the higher levels of approximation.

Substituting (4.16) into (4.10)-(4.12) yields

$$
w_{1 x x}=D_{0}^{2} w_{1}+2 i \sum_{s}^{\infty} \omega_{s} D_{1} A_{s} \frac{\sin \omega_{s} x}{\sin \omega_{s}} e^{i \omega_{s} T_{0}}+c c
$$




$$
\begin{array}{cc}
w_{1}=0 & \text { at } x=0 \\
D_{0}^{2} w_{1}+\alpha_{1} w_{1 x}+\alpha_{4} w_{1}= & -2 i \sum_{s=1}^{\infty} \omega_{s} D_{1} A_{s} e^{i \omega_{s} T_{0}} \\
& -\alpha_{5}\left[\sum_{s, m}^{\infty} A_{s} A_{m} e^{l\left(\omega_{s}+\omega_{m}\right) T_{0}}\right. \\
& \left.+\sum_{s, m}^{\infty} A_{s} \bar{A}_{m} e^{i\left(\omega_{s}-\omega_{m}\right) T_{0}}\right] \\
+c c & \text { at } x=1
\end{array}
$$

The solvability condition of (4.18)-(4.20) demands that

$$
D_{1} A_{s}=0
$$

if there are no internal resonances of the form $\omega_{\mathrm{s}} \cong 2 \omega_{\mathrm{m}}$ or $\omega_{\mathrm{s}} \cong \omega_{\mathrm{m}}+\omega_{\mathrm{k}}$. Substituting (4.21) into (4.18)-(4.20) and solving for $w_{1}$ yields

$$
\begin{aligned}
& w_{1}=\sum_{s, m}^{\infty} C_{1} A_{s} A_{m} \frac{\sin \left(\omega_{s}+\omega_{m}\right) x}{\sin \left(\omega_{s}+\omega_{m}\right)} e^{i\left(\omega_{s}+\omega_{m}\right) T_{0}} \\
& +\sum_{s, m}^{\infty} C_{2} A_{s} \bar{A}_{m} \frac{\sin \left(\omega_{s}-\omega_{m}\right) x}{\sin \left(\omega_{s}-\omega_{m}\right)} e^{i\left(\omega_{s}-\omega_{m}\right) T_{0}}+c c
\end{aligned}
$$

where

$$
C_{1}=\frac{-\alpha_{5} \sin \left(\omega_{s}+\omega_{m}\right)}{\alpha_{1}\left(\omega_{s}+\omega_{m}\right) \cos \left(\omega_{s}+\omega_{m}\right)+\left[\alpha_{4}-\left(\omega_{s}+\omega_{m}\right)^{2}\right] \sin \left(\omega_{s}+\omega_{m}\right)}
$$

and 


$$
\mathrm{C}_{2}=\frac{-\alpha_{5} \sin \left(\omega_{s}-\omega_{m}\right)}{\alpha_{1}\left(\omega_{s}-\omega_{m}\right) \cos \left(\omega_{s}-\omega_{m}\right)+\left[\alpha_{4}-\left(\omega_{s}-\omega_{m}\right)^{2}\right] \sin \left(\omega_{s}-\omega_{m}\right)}
$$

Substituting (4.16), (4.21), and (4.22) into (4.13)-(4.15) yields

$$
\begin{aligned}
& \mathrm{w}_{2 \mathrm{xx}}=\mathrm{D}_{0}^{2} \mathrm{w}_{2}+2 \mathrm{i} \sum_{s=1}^{\infty} \omega_{s}\left(\mathrm{~A}_{s}^{\prime}+\mu \mathrm{A}_{s}\right) \frac{\sin \omega_{s} \mathrm{x}}{\sin \omega_{s}} \mathrm{e}^{\mathrm{i} \omega_{s} \mathrm{~T}_{0}}+c c \\
& \mathrm{w}_{2}=0 \quad \text { at } \quad \mathrm{x}=0 \\
& D_{0}^{2} w_{2}+\alpha_{1} w_{2 x}+\alpha_{4} w_{2}=\frac{1}{2} f_{1} e^{\left(\omega_{s} T_{0}+\sigma T_{2}\right)}-2 i \sum_{s=1}^{\infty} \omega_{s} A^{\prime}{ }_{s} e^{i \omega_{s} T_{0}} \\
& -2 \alpha_{5}\left[\sum_{s, m, k}^{\infty} C_{1} A_{s} A_{m} A_{k} e^{i\left(\omega_{s}+\omega_{m}+\omega_{k}\right) T_{0}}\right. \\
& \left.+\sum_{s, m, k}^{\infty}\left(C_{1}+2 C_{2}\right) A_{s} A_{m} \bar{A}_{k} e^{i\left(\omega_{s}+\omega_{m}-\omega_{k}\right) T_{0}}\right] \\
& -\alpha_{3}\left[\sum_{s, m, k}^{\infty} A_{s} A_{m} A_{k} e^{i\left(\omega_{s}+\omega_{m}+\omega_{k}\right) T_{0}}\right. \\
& \left.+3 \sum_{s, m, k}^{\infty} A_{s} A_{m} \bar{A}_{k} e^{i\left(\omega_{s}+\omega_{m}-\omega_{k}\right) T_{0}}\right] \\
& +c c \\
& \text { at } \mathrm{x}=1
\end{aligned}
$$

To determine the solution of (4.25)-(4.27), we use the principle of superposition and write

$$
w_{2}=\sum_{s=1}^{\infty} \varphi_{s}\left(x, T_{2}\right) e^{i \omega_{s} T_{0}}+W_{2}\left(x, T_{0}, T_{2}\right)+c c
$$

where $\mathrm{W}_{2}$ is governed by equations (4.25)-(4.27) with the terms proportional to $\exp \left(i \omega_{3} T_{0}\right)$ being deleted. Therefore $W_{2}$ exists, is unique, and is free of small-divisor 
terms. Substituting (4.28) into (4.25)-(4.27) and equating the coefficients of $\exp \left(i \omega_{8} T_{0}\right)$ on both sides of each equation, we obtain

$$
\begin{gathered}
\varphi^{\prime \prime}{ }_{s}+\omega_{s}^{2} \varphi_{s}=2 i \omega_{s}\left(A_{s}^{\prime}+\mu A_{s}\right) \frac{\sin \omega_{s} x}{\sin \omega_{s}} \\
\varphi_{s}=0 \quad \text { at } x=0 \\
\varphi_{s}^{\prime}+\left(\frac{\alpha_{4}-\omega_{s}^{2}}{\alpha_{1}}\right) \varphi_{s}=\frac{1}{\alpha_{1}}\left[\frac{1}{2} \delta_{n s} f_{1} e^{i \sigma T_{2}}-2 i \omega_{s} A_{s}^{\prime}\right. \\
\left.-2 \alpha_{5} B\left(A_{s}^{2} \bar{A}_{s}+2 A_{s} \sum_{r \neq s} A_{r} \bar{A}_{r}\right)\right] \text { at } x=1
\end{gathered}
$$

where $\delta_{\mathrm{m}}$ is the kronecker delta and the constant $B$ is given by

$$
B=C_{1}+2 C_{2}+\frac{3 \alpha_{3}}{2 \alpha_{5}}
$$

Since the homogeneous equations (4.29)-(4.31) have a non-trivial solution, the inhomogeneous equations have a solution only if the solvability conditions are satisfied [10]. Following the procedure outlined in Appendix A leads to the following solvability conditions:

$$
\begin{aligned}
2 i \omega_{s}\left(A^{\prime} s\right. & \left.+\mu_{s} A_{s}\right)=-\frac{\Gamma_{s}}{\alpha_{1}}\left[2 i \omega_{s} A_{s}^{\prime}+2 \alpha_{5} B\left(A_{s}^{2} \bar{A}_{s}+2 A_{s} \sum_{r \neq s} A_{r} \bar{A}_{r}\right)\right] \text {, for } s \neq n \\
2 i \omega_{n}\left(A_{n}^{\prime}+\mu_{n} A_{n}\right)=-\frac{\Gamma_{n}}{\alpha_{1}}\left[2 i \omega_{n} A_{n}^{\prime}\right. & \\
& \left.+\alpha_{5} B\left(A_{n}^{2} \bar{A}_{n}+2 A_{n} \sum_{r \neq n} A_{r} \bar{A}_{r}-\frac{1}{2} f_{1} e^{l \sigma T_{2}}\right)\right]
\end{aligned}
$$

where 


$$
\mu_{k}=\frac{\Gamma_{k}}{\sin ^{2} \omega_{k}} \int_{0}^{1} \mu(x) \sin ^{2} \omega_{k} x d x
$$

Expressing the $A_{k}$ in the polar form

$$
A_{k}=\frac{1}{2} a_{k}\left(T_{2}\right) \exp \left[i \beta_{k}\left(T_{2}\right)\right]
$$

where $a_{k}$ and $\beta_{k}$ are real functions representing the amplitudes and phases, respectively. Then, substituting (4.37) into (4.33) and (4.34) and separating each equation into real and imaginary parts yields

$$
\begin{gathered}
\dot{a}_{s}=-\frac{\alpha_{1} \mu_{s}}{\alpha_{1}+\Gamma_{s}} a_{s} \\
\omega_{s}\left(\frac{\alpha_{1}+\Gamma_{s}}{\alpha_{1}}\right) a_{s} \dot{\beta}_{s}=\frac{\Gamma_{s} \alpha_{5}}{4 \alpha_{1}} B\left(a_{s}^{3}+2 a_{s} \sum_{r \neq s} a_{r}^{2}\right) \text { for } s \neq n
\end{gathered}
$$

and

$$
\begin{gathered}
\left(\frac{\alpha_{1}+\Gamma_{n}}{\alpha_{1}}\right) \omega_{n} \dot{a}_{n}=-\omega_{n} \mu_{n} a_{n}+\frac{\Gamma_{n} f_{1}}{2 \alpha_{1}} \sin \gamma \\
\omega_{n}\left(\frac{\alpha_{1}+\Gamma_{n}}{\alpha_{1}}\right) a_{n} \dot{\beta}_{n}=\frac{\Gamma_{n}}{\alpha_{1}}\left[\frac{1}{4} \alpha_{5} B a_{n}^{3}+\frac{1}{2} \alpha_{5} B a_{n} \sum_{r \neq n} a_{r}^{2}-\frac{1}{2} f_{1} \cos \gamma\right] \text { for } s=n(4.41)
\end{gathered}
$$

where

$$
\gamma=\sigma T_{2}-\beta_{n}
$$

It follows from (4.38) that $a_{8} \propto \exp \left(-\frac{\alpha_{1} \mu_{8} T_{2}}{\alpha_{1}+\Gamma_{3}}\right)$ and hence all $a_{3}$, except $a_{n}$, decay. 
To determine the character of the solution to the system of equations (4.40) and (4.41), one needs to first locate the singular points, often referred to as equilibrium or fixed points, and then examine the motion in the neighborhood of these points. The singular points correspond to constant values of $a_{n}$ and $\gamma$. Hence, the response of the system is said to be a steady-state motion. When the phase trajectories are plotted in the neighborhood of the equilibrium points, they show whether a small perturbation to the steady-state motion grows or decays. Letting $a=0$ and $\dot{\gamma}=0$ in (4.40) and (4.41) yields

$$
\begin{gathered}
\omega_{n} \mu_{n} a_{n}=\frac{\Gamma_{n} f_{1}}{2 \alpha_{1}} \sin \gamma \\
\omega_{n}\left(\frac{\alpha_{1}+\Gamma_{n}}{\alpha_{1}}\right) a_{n} \sigma-\frac{\Gamma_{n} \alpha_{5}}{4 \alpha_{1}} B a_{n}^{3}=-\frac{\Gamma_{n} f_{1}}{2 \alpha_{1}} \cos \gamma
\end{gathered}
$$

Eliminating $\gamma$ from equations (4.43) and (4.44) yields

$$
\left(\frac{\alpha_{1}+\Gamma_{n}}{\alpha_{1}}\right) \omega_{n} \sigma=\frac{\Gamma_{n} \alpha_{5}}{4 \alpha_{1}} B a_{n}^{2} \mp\left[\frac{\Gamma_{n}^{2} f_{1}^{2}}{4 \alpha_{1}^{2} a_{n}^{2}}-\omega_{n}^{2} \mu_{n}^{2}\right]^{1 / 2}
$$

Using (4.45), we plot the amplitude of the steady-state solution in figure 3 against the detuning parameter $\sigma$. In the same way, a representative variation of the steady-state amplitude with the excitation amplitude is shown in figure 4.

To determine the stability of the steady-state solutions, we perturb these solutions by letting

$$
a=a_{0}+a_{1}\left(T_{2}\right)
$$




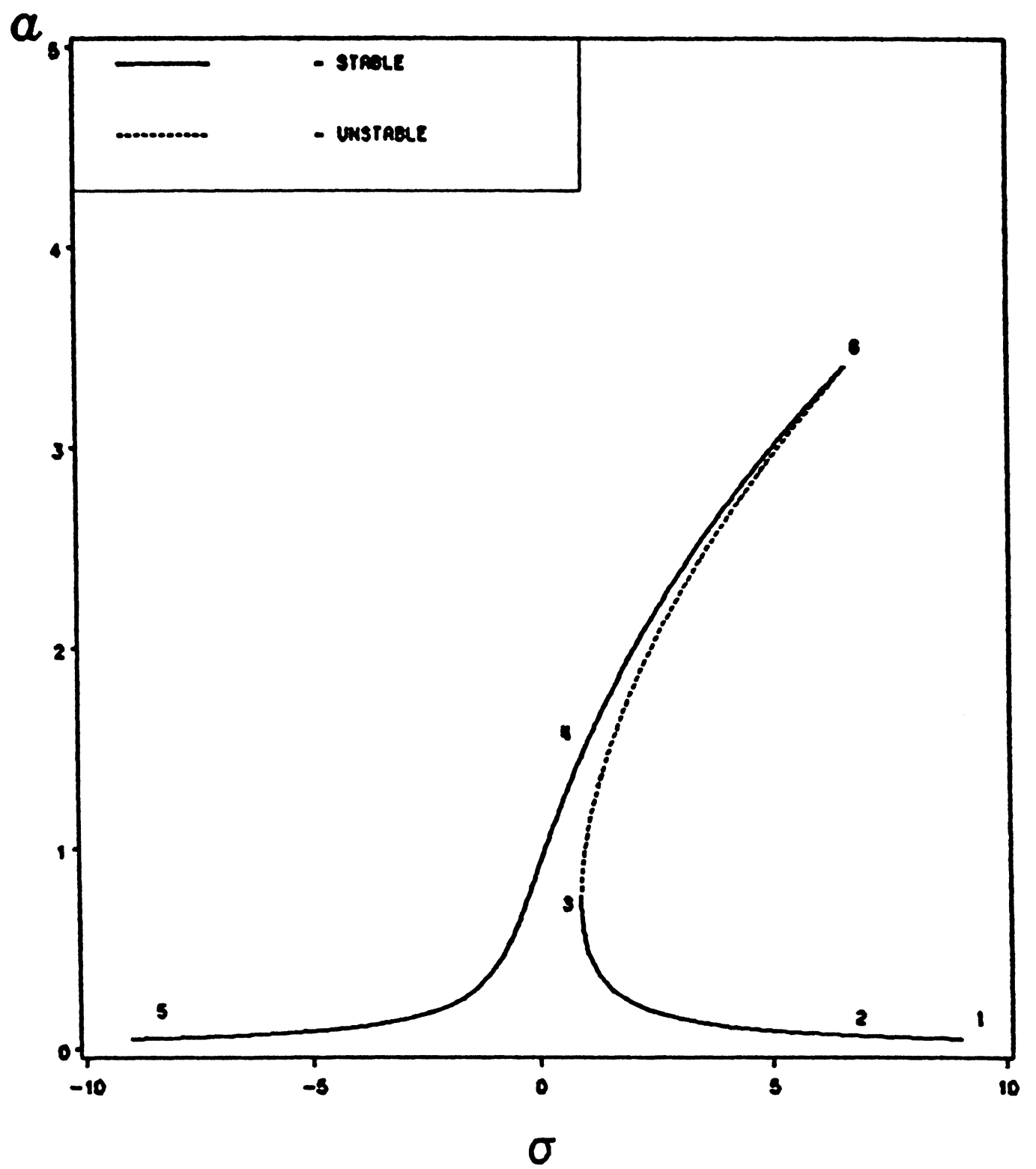

Figure 3. A representative frequency-response curve for the case of primary resonance $\left(\alpha_{1}=2, \alpha_{2}=20, \alpha_{3}=-4, f_{0}=20, f_{1}=25, \mu=.15\right)$ 


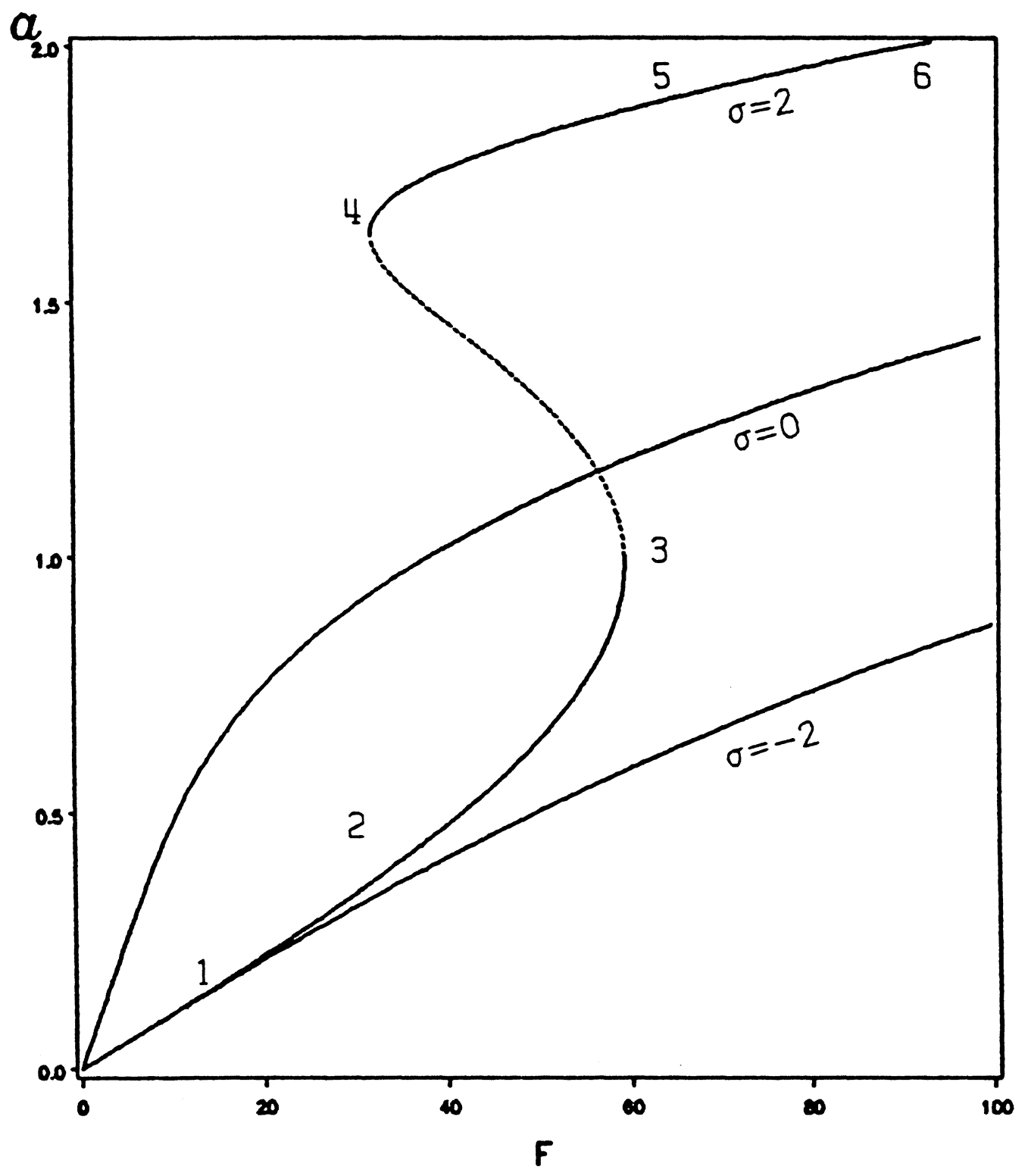

Figure 4. Representative variations of the response amplitude with the excitation amplitude for several detunings, primary resonance $\left(\alpha_{1}=2, \alpha_{-} 20, \alpha_{3}=-7, f_{0}=20, \mu=.5\right)$ 


$$
\gamma=\gamma_{0}+\gamma_{1}\left(T_{2}\right)
$$

where $a_{0}$ and $\gamma_{0}$ correspond to a given steady-state. Substituting (4.46) and (4.47) into (4.40) and (4.41) and linearizing the result, we obtain

$$
\left\{\begin{array}{l}
\dot{a}_{1} \\
\dot{\gamma}_{1}
\end{array}\right\}=[A]\left\{\begin{array}{l}
a_{1} \\
\gamma_{1}
\end{array}\right\}
$$

where the matrix $A$ is defined according to

$$
[A]=\left[\begin{array}{cc}
\frac{-\mu_{n} \alpha_{1}}{\alpha_{1}+\Gamma_{n}} & -a_{0} \sigma+\frac{\Gamma_{n} \alpha_{5} B a_{0}^{3}}{\omega_{n}\left(\alpha_{1}+\Gamma_{n}\right)} \\
\frac{\sigma}{a_{0}} & \frac{\alpha_{1} \alpha_{5} \mu_{n} B}{f_{1}\left(\alpha_{1}+\Gamma_{n}\right)}
\end{array}\right]
$$

For the solution around a fixed point to be asymptotically stable, the eigenvalues of the matrix $[A]$ must have negative real parts. A stability analysis has been carried out for the steady-state solutions in figures 3 and 4 ; the unstable solutions are represented by the dashed lines whereas the stable solutions are represented by the solid lines. The multi-valuedness of the solutions in these figures leads to the jump phenomenon. Its physical significance is best described by the following example [1]. Suppose that

an experiment is performed where the amplitude of the excitation is held constant while slowly varying the detuning parameter $\sigma$ and observing the variation in the response amplitude, then the curve obtained while increasing the detuning will not be exactly the same as that obtained while decreasing the detuning. For example, if one starts at point 5 on figure 3 and slowly increases the detuning, the response ampli- 
tude will also increase up to point 6 where a downward jump to point 2 occurs. Thereafter, the response amplitude will slowly decrease as the detuning is increased. Now suppose one starts at point 1 and slowly decreases the detuning, the response amplitude will slowly increase up to point 3 where an upward jump occurs from point 3 to point 4 . A further decrease in the detuning will result in a decrease in the response amplitude. For positive values of the detuning $\sigma$, the same jump phenomenon is observed in figure 4. For instance, consider the curve $\sigma=2$, as one increases the excitation amplitude from that corresponding to point 1 the response amplitude also increases until point 3 (the tangent to the curve becomes vertical). As the excitation amplitude increases further, the response jumps vertically upward to point 5. Further increases in $F$ produces slow increases in a. The same thing happens when the excitation amplitude is decreased from that corresponding to point 6 , a decreases past point 5. As $F$ decreases beyond point 4 , a jumps downward to point 2 and the response slowly decreases along the curve 3-2-1.

\subsection{Method of harmonic balance}

Substituting (4.16) and (4.22) into (3.12), considering only the nth mode of vibration, using (3.1), (3.6), (4.37), and (4.42), and putting $\varepsilon=1$, we obtain the approximate solution

$$
u \cong\left(b+\frac{1}{4} C_{2} a_{n}^{2}\right) x+a_{n} \frac{\sin \omega_{n} x}{\sin \omega_{n}} \cos (\Omega t-\gamma)+\frac{1}{4} C_{1} a_{n}^{2} \frac{\sin 2 \omega_{n} x}{\sin 2 \omega_{n}} \cos (2 \Omega t-2 \gamma)(4.50)
$$


Thus, to the second approximation, u consists of three terms: a static term, a term having a frequency equal to the excitation frequency, and a term having a frequency equal to twice the excitation frequency. Consequently, in order to obtain a consistent approximation using the method of harmonic balance, one needs to assume a solution having the form in (4.50). Dokainish and Elmadany assumed a solution that does not contain the second harmonic and hence obtained results with limited validity. In fact, using the form (4.50) as in Appendix B, we obtain the following nonlinearity coefficient $\beta_{\bullet}$ :

$$
\beta_{\mathrm{e}}=\frac{3}{4} \beta\left(1-\alpha_{\mathrm{e}}\right)
$$

where $\alpha_{0}$ is the non-linear contribution due to the second harmonic. Figure 12 shows the variation of $\alpha_{0}$ with $b$, the response amplitude due to the constant pressure $\Phi_{0}$ in (2.10). For small $b$, that is small $\Phi_{0}, \alpha_{0}$ is small and can be neglected, and hence the results obtained by Dokainish and Elmadany are valid. However, for large b, that is large $\Phi_{0}$, which is usually the case, $\alpha_{0}$ is no longer negligible. In fact, the non-linear

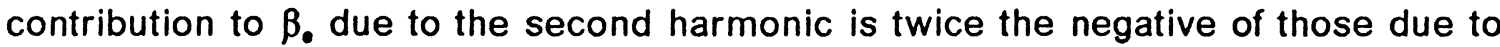
the first harmonic alone. For values of $\Phi_{0}$ that are neither small nor large, a correction $\alpha_{0}$ to $\beta_{0}$ may or may not be obtained depending on the value of the constant $C$ given by equation (B15). The singularity in $\beta_{0}$ is due to the two-to-one internal resonance. 


\section{Case of Subharmonic resonance of order $1 / 3$}

This type of resonance occurs whenever the excitation frequency $\Omega$ is near three times the value of one of the linear natural frequencies $\omega_{n}$. To describe the nearness of this resonance, one introduces a detuning parameter $\sigma$ defined according to

$$
\Omega=3 \omega_{n}+\varepsilon^{2} \sigma
$$

Ordering for this case is accomplished in a manner so that the excitation appears at the lowest order in the perturbation equations.

Letting

$$
\lambda=1, \quad \mu_{1}=\varepsilon \mu, \quad \frac{F_{1}}{\varepsilon^{\lambda}}=f_{1}
$$

and substituting (5.2) into (3.7)-(3.9), one obtains

$$
w_{x x}=w_{t t}+2 \varepsilon^{2} \mu w_{t}
$$

$w=0$

at $x=0$ 


$$
w_{t t}+\alpha_{1} w_{x}+\varepsilon \alpha_{5} w^{2}+\varepsilon^{2} \alpha_{3} w^{3}=f_{1} \cos \Omega t \quad \text { at } x=1
$$

Substituting (3.12) into (5.3)-(5.5) and equating coefficients of like powers of $\varepsilon$ on both sides of each equation, one obtains linear problems that can be solved in succession. The zeroth-order problem is

$$
\begin{array}{cc}
w_{0 x x}=D_{0}^{2} w_{0} & \\
w_{0}=0 & \text { at } x=0 \\
D_{0}^{2} w_{0}+\alpha_{1} w_{0 x}+\alpha_{4} w_{0}=f_{1} \cos \Omega T_{0} & \text { at } x=1
\end{array}
$$

Since in the absence of internal resonances all modes that are not directly excited decay with time, we include only the nth mode and express the solution of (5.6)-(5.8) as

$$
w_{0}=A_{n} \frac{\sin \omega_{n} X}{\sin \omega_{n}} e^{i \omega_{n} T_{0}}+\Lambda \frac{\sin \Omega x}{\sin \Omega} e^{i \Omega T_{0}}+c c
$$

where $\omega_{n}$ is defined by (4.17) and

$$
\Lambda=\frac{1}{2} \frac{f_{1} \sin \Omega}{\left(\alpha_{4}-\Omega^{2}\right) \sin \Omega+\alpha_{1} \Omega \cos \Omega}
$$

The first-order problem is

$$
\begin{gathered}
w_{1 x x}=D_{0}^{2} w_{1}+2 D_{0} D_{1} w_{0} \\
w_{1}=0 \quad \text { at } x=0
\end{gathered}
$$




$$
D_{0}^{2} w_{1}+\alpha_{1} w_{1 x}+\alpha_{4} w_{1}=-2 D_{0} D_{1} w_{0}-\alpha_{5} w_{0}^{2} \text { at } x=1
$$

Substituting (5.9) into (5.11)-(5.13), one finds that the solvability condition is $D_{1} A=0$. Then, the solution is

$$
\begin{aligned}
w_{1}= & C_{1} A_{n}^{2} \frac{\sin 2 \omega_{n} x}{\sin 2 \omega_{n}} e^{2 i \omega_{n} T_{0}}+C_{2} \Lambda^{2} \frac{\sin 2 \Omega_{n} x}{\sin 2 \Omega_{n}} e^{2 i \Omega T_{0}} \\
& +C_{3} \Lambda A_{n} \frac{\sin \left(\omega_{n}+\Omega\right) x}{\sin \left(\omega_{n}+\Omega\right)} e^{i\left(\omega_{n}+\Omega\right) T_{0}}+C_{4} \Lambda \bar{A}_{n} \frac{\sin \left(\Omega-\omega_{n}\right) x}{\sin \left(\Omega-\omega_{n}\right)} e^{i\left(\Omega-\omega_{n}\right) T_{0}} \\
& -\frac{\alpha_{5}}{\alpha_{1}+\alpha_{4}}\left(A_{n} \bar{A}_{n}+\Lambda^{2}\right) x+c c
\end{aligned}
$$

where

$$
\begin{gathered}
C_{1}=\frac{-\alpha_{5} \sin 2 \omega_{n}}{2 \alpha_{1} \omega_{n} \cos 2 \omega_{n}+\left(\alpha_{4}-4 \omega_{n}^{2}\right) \sin 2 \omega_{n}} \\
C_{2}=\frac{-\alpha_{5} \sin 2 \Omega}{2 \alpha_{1} \Omega \cos 2 \Omega+\left(\alpha_{4}-4 \Omega^{2}\right) \sin 2 \Omega} \\
C_{3}=\frac{-2 \alpha_{5} \sin \left(\omega_{n}+\Omega\right)}{\alpha_{1}\left(\omega_{n}+\Omega\right) \cos \left(\omega_{n}+\Omega\right)+\left[\alpha_{4}-\left(\omega_{n}+\Omega\right)^{2}\right] \sin \left(\omega_{n}+\Omega\right)} \\
C_{4}=\frac{-2 \alpha_{5} \sin \left(\Omega-\omega_{n}\right)}{\alpha_{1}\left(\Omega-\omega_{n}\right) \cos \left(\Omega-\omega_{n}\right)+\left[\alpha_{4}-\left(\Omega-\omega_{n}\right)^{2}\right] \sin \left(\Omega-\omega_{n}\right)}
\end{gathered}
$$

Since the $A_{n}$ are independent of $T_{1}$, the second-order problem can be written as

$$
\begin{aligned}
& w_{2 x x}=D_{0}^{2} w_{2}+2 D_{0} D_{2} w_{0}+2 \mu D_{0} w_{0} \\
& w_{2}=0 \quad \text { at } x=0
\end{aligned}
$$




$$
D_{0}^{2} w_{2}+\alpha_{1} w_{2 x}+\alpha_{4} w_{2}=-2 D_{0} D_{2} w_{0}-2 \alpha_{5} w_{0} w_{1}-\alpha_{3} w_{0}^{3} \text { at } x=1
$$

To determine the solvability condition of (5.19)-(5.21), we substitute for $w_{0}$ and $w_{1}$, let $w_{2}=\varphi_{n} \exp \left(i \omega_{n} T_{0}\right)$, equate the coefficients of $\exp \left(i \omega_{n} T_{0}\right)$ on both sides, and obtain

$$
\begin{aligned}
\varphi_{n}^{\prime \prime} & +\omega_{n}^{2} \varphi_{n}=2 i \omega_{n}\left(A_{n}^{\prime}+\mu_{n} A_{n}\right) \frac{\sin \omega_{n} x}{\sin \omega_{n}} \\
\varphi_{n} & =0 \quad \text { at } \quad x=0 \\
\alpha_{1} \varphi_{n}^{\prime}+\left(\alpha_{4}-\omega_{n}^{2}\right) \varphi_{n} & =-2 i \omega_{n} A_{n}^{\prime} \\
& -2 \alpha_{5}\left[B_{1} A_{n}^{2} \bar{A}_{n}+B_{2} \bar{A}_{n}^{2} \Lambda e^{l \sigma T_{2}}+B_{3} A_{n} \Lambda^{2}\right] \text { at } x=1
\end{aligned}
$$

where $\mu_{n}$ is defined in (4.36) and

$$
\begin{gathered}
\mathrm{B}_{1}=\mathrm{C}_{1}-\frac{2 \alpha_{5}}{\alpha_{1}+\alpha_{4}}+\frac{3 \alpha_{3}}{2 \alpha_{5}} \\
\mathrm{~B}_{2}=\mathrm{C}_{1}+\mathrm{C}_{4}+\frac{3 \alpha_{3}}{2 \alpha_{5}} \\
\mathrm{~B}_{3}=\mathrm{C}_{3}+\mathrm{C}_{4}-\frac{2 \alpha_{5}}{\alpha_{1}+\alpha_{4}}+\frac{3 \alpha_{3}}{\alpha_{5}}
\end{gathered}
$$

The solvability condition for equations (5.22)-(5.24) can be determined as in Appendix A. The result is

$$
2 i \omega_{n}\left(A_{n}^{\prime}+\mu_{n} A_{n}\right)=-\frac{\Gamma_{n}}{\alpha_{1}}\left[2 i \omega_{n} A_{n}^{\prime}+2 \alpha_{5}\left(B_{1} A_{n}^{2} \bar{A}_{n}+B_{2} \bar{A}_{n}^{2} \Lambda e^{l \sigma T_{2}}+B_{3} A_{n} \Lambda^{2}\right)\right]
$$

where $\Gamma_{n}$ is defined in (4.35). 
Expressing $A_{n}$ in the polar form (4.37) and separating (5.28) into real and imaginary parts yields

$$
\begin{gathered}
\left(\frac{\alpha_{1}+\Gamma_{n}}{\alpha_{1}}\right) \omega_{n} \dot{a}_{n}=-\omega_{n} \mu_{n} a_{n}-\frac{\Gamma_{n} \alpha_{5}}{2 \alpha_{1}} B_{2} \Lambda a_{n}^{2} \sin \gamma \\
\omega_{n}\left(\frac{\alpha_{1}+\Gamma_{n}}{\alpha_{1}}\right) a_{n} \dot{\beta}_{n}=\frac{\Gamma_{n} \alpha_{5}}{\alpha_{1}}\left[\frac{1}{4} B_{1} a_{n}^{3}+\frac{1}{2} B_{2} a_{n}^{2} \Lambda \cos \gamma+B_{3} a_{n} \Lambda^{2}\right]
\end{gathered}
$$

where

$$
\gamma=\sigma T_{2}-3 \beta_{n}
$$

It follows from equations (5.29) and (5.30) that the steady-state motion corresponds to $\mathrm{a}=0$ and $\dot{\gamma}=0$. The steady-state amplitude and phase are given by

$$
\begin{gathered}
\omega_{n} \mu_{n} a_{n}=-\frac{\Gamma_{n} \alpha_{5}}{2 \alpha_{1}} B_{2} \Lambda a_{n}^{2} \sin \gamma \\
\frac{1}{3} \omega_{n}\left(\frac{\alpha_{1}+\Gamma_{n}}{\alpha_{1}}\right) a_{n} \sigma-\frac{\Gamma_{n} \alpha_{5}}{\alpha_{1}}\left(\frac{1}{4} B_{1} a_{n}^{3}+B_{3} a_{n} \Lambda^{2}\right)=\frac{\Gamma_{n} \alpha_{5}}{2 \alpha_{1}} B_{2} a_{n}^{2} \Lambda \cos \gamma
\end{gathered}
$$

Eliminating $\gamma$ from equations (5.32) and (5.33) yields

$$
\begin{aligned}
\frac{\Gamma_{n}^{2} \alpha_{5}^{2} B_{2}^{2} a_{n}^{4} \Lambda^{2}}{4 \alpha_{1}^{2}}= & \omega_{n}^{2} \mu_{n}^{2} a_{n}^{2} \\
& +\left[\frac{1}{3} \omega_{n}\left(\frac{\alpha_{1}+\Gamma_{n}}{\alpha_{1}}\right) a_{n} \sigma-\frac{\Gamma_{n} \alpha_{5}}{\alpha_{1}}\left(\frac{1}{4} B_{1} a_{n}^{3}+B_{3} a_{n} \Lambda^{2}\right)\right]^{2}
\end{aligned}
$$

It follows from equation (5.34) that either $a_{n}=0$ or 


$$
\begin{aligned}
\frac{1}{3} \omega_{n}\left(\frac{\alpha_{1}+\Gamma_{n}}{\alpha_{1}}\right) \sigma= & \frac{\Gamma_{n} \alpha_{5}}{\alpha_{1}}\left(\frac{1}{4} B_{1} a_{n}^{2}+B_{3} \Lambda^{2}\right) \\
& \overline{+}\left[\frac{\Gamma_{n}^{2} \Lambda^{2} \alpha_{5}^{2} B_{2}^{2} a_{n}^{2}}{4 \alpha_{1}^{2}}-\omega_{n}^{2} \mu_{n}^{2}\right]^{1 / 2}
\end{aligned}
$$

A representative frequency-response curve is shown in figure 5. Note that although the frequency of the excitation is three times the natural frequency of the system, the response can be quite large. Jump phenomenon is not present in this case. Unlike linear systems, in the region where two stable solutions exist $\left(a_{n}=0, a_{n} \neq 0\right)$, the initial conditions determine which solution represents the response. Figure 6 shows the variation of the response amplitude with the excitation amplitude for three different values of the detuning $\sigma$. It shows that subharmonic resonances cannot be excited for values of the excitation amplitude outside a certain range and may or may not be excited for values within that range. It also shows that for this type of resonance increasing the excitation amplitude may cause a decrease rather than an increase in the response amplitude.

Dokainish and Elmadany analyzed this case using the method of harmonic balance and assumed the following form for the solution :

$$
u=A x+B_{1} \sin \lambda x \sin \Omega t+B_{3} \sin 3 \lambda x \sin 3 \Omega t
$$

However, equations (5.9) and (5.14) reveal that, in order to obtain a consistent solution, the second harmonic must be included in the analysis. Therefore, assuming the following solution for $u$ :

$$
u=A x+B_{1} \sin \lambda x \sin \Omega t+B_{2} \sin 2 \lambda x \sin 2 \Omega t+B_{3} \sin 3 \lambda x \sin 3 \Omega t
$$




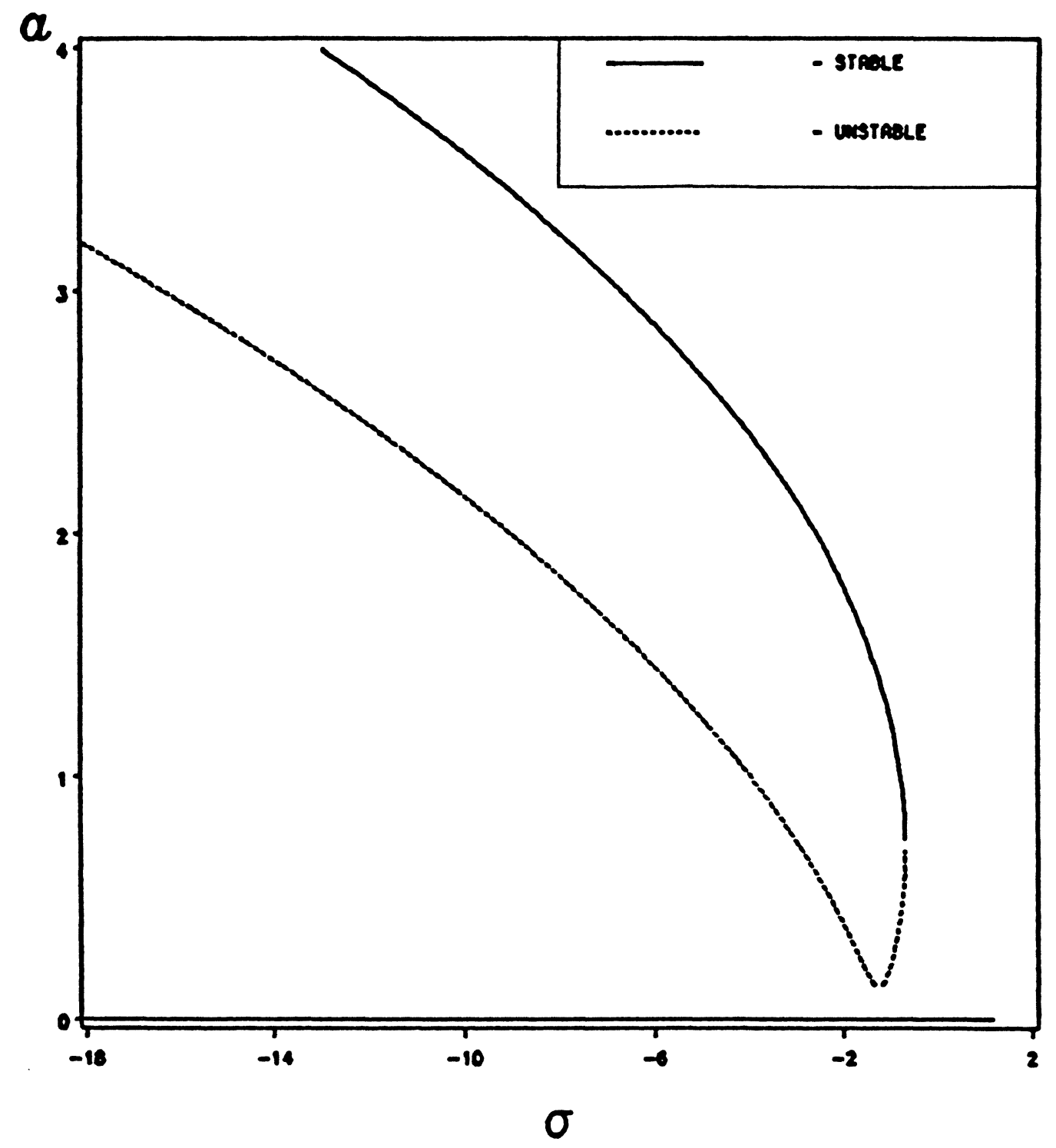

Flgure 5. Frequency-response curve for the case of subharmonic resonance of order one-third $\left(\alpha_{1}=2, \alpha_{2}=20, \alpha_{3}=3, f_{0}=10, f_{1}=20, \mu=.08\right)$ 


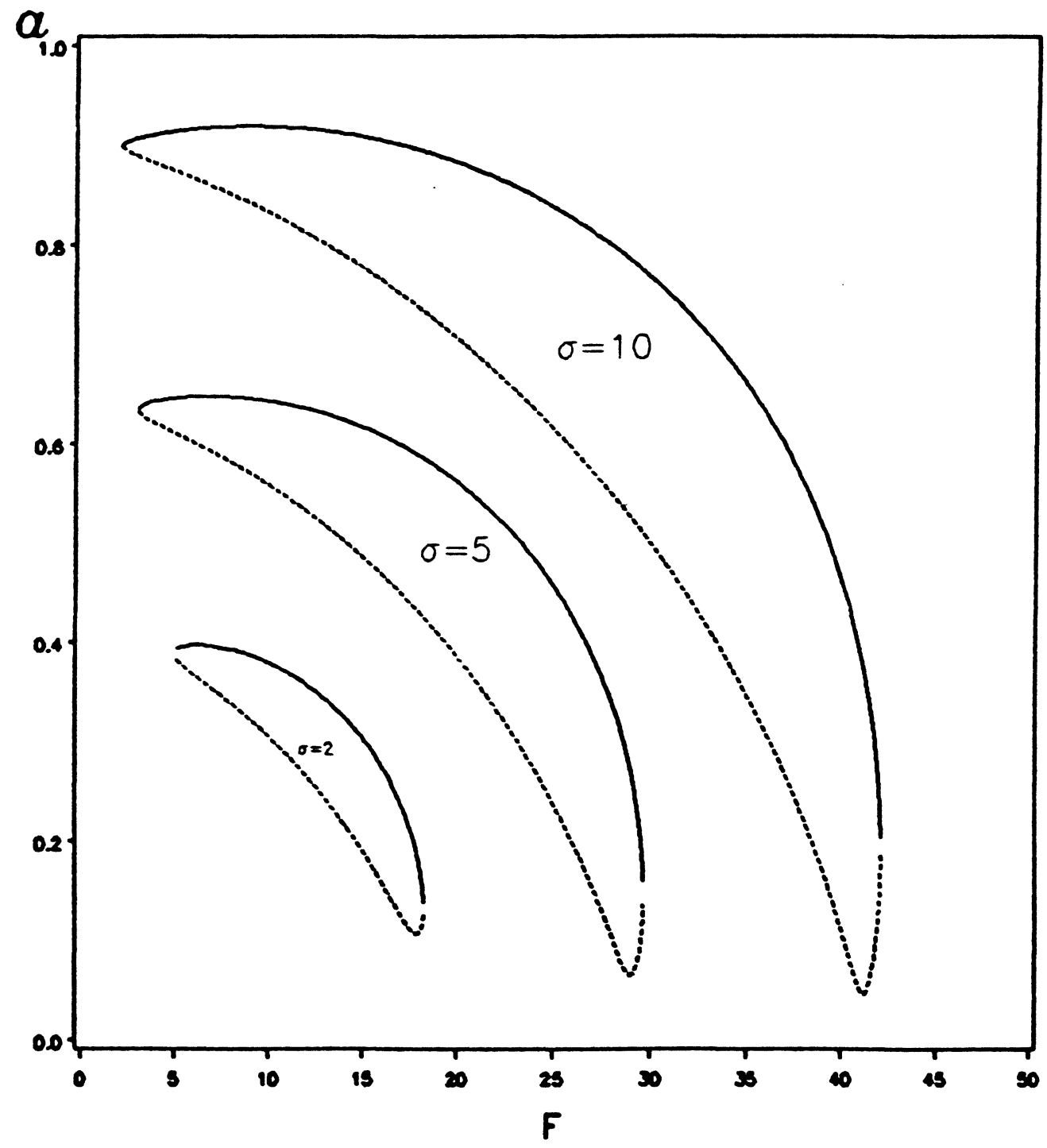

Figure 6. Response amplitude as a function of the excitation amplitude for different values of the detuning: Case of subharmonic resonance of order one-third $\left(a_{1}=2, a_{2}=20, a_{3}=-5, f_{0}=10, \mu=.08\right)$ 
and using the method of harmonic balance as shown in Appendix B, one can show that the Dokainish-Elmadany results are valid only for small static pressures. 


\section{Case of Superharmonic resonance of order 3}

To express the nearness of this resonance, we introduce the detuning parameter $\sigma$ defined according to

$$
3 \Omega=\omega_{n}+\varepsilon^{2} \sigma
$$

Equations (5.6) through (5.23) hold in this case $\Omega \cong \frac{1}{3} \omega_{n}$ and (5.24) is replaced with

$$
\alpha_{1} \varphi_{n}^{\prime}+\left(\alpha_{4}-\omega_{n}^{2}\right) \varphi_{n}=-2 i \omega_{n} A_{n}^{\prime}-2 \alpha_{5}\left[B_{1} A_{n}^{2} \bar{A}_{n}+B_{4} \Lambda^{3} e^{i \sigma T_{2}}+B_{3} A_{n} \Lambda^{2}\right] \quad \text { at } \quad x=1
$$

where the constants $B_{1}$ and $B_{3}$ are given by equations (5.9) and (5.10), respectively, whereas $B_{4}$ is defined according to

$$
B_{4}=C_{2}+\frac{\alpha_{3}}{2 \alpha_{5}}
$$

where $C_{2}$ is defined in (5.16). The solvability condition for (6.2) is determined in the same manner as outlined in Appendix $A$. The result is 


$$
2 i \omega_{n}\left(A_{n}^{\prime}+\mu_{n} A_{n}\right)=-\frac{\Gamma_{n}}{\alpha_{1}}\left[2 i \omega_{n} A_{n}^{\prime}+2 \alpha_{5}\left(B_{1} A_{n}^{2} \bar{A}_{n}+B_{4} \Lambda^{3} e^{i \sigma T_{2}}+B_{3} A_{n} \Lambda^{2}\right)\right]
$$

where $\Gamma_{n}$ and $\mu_{n}$ are defined in equations (4.35) and (4.36), respectively.

Substituting the polar form (4.37) into (6.4) and separating into real and imaginary parts, one obtains

$$
\begin{gathered}
\left(\frac{\alpha_{1}+\Gamma_{n}}{\alpha_{1}}\right) \omega_{n} \dot{a}_{n}=-\omega_{n} \mu_{n} a_{n}-\frac{2 \Gamma_{n} \alpha_{5}}{\alpha_{1}} B_{4} \Lambda^{3} \sin \gamma \\
\omega_{n}\left(\frac{\alpha_{1}+\Gamma_{n}}{\alpha_{1}}\right) a_{n} \dot{\beta}_{n}=\frac{\Gamma_{n} \alpha_{5}}{\alpha_{1}}\left(\frac{1}{4} B_{1} a_{n}^{3}+B_{3} a_{n} \Lambda^{2}+2 B_{4} \Lambda^{3} \cos \gamma\right)
\end{gathered}
$$

where

$$
\gamma=\sigma T_{2}-\beta_{n}
$$

The steady-state motion can be determined by letting $a=\dot{\gamma}=0$ in (6.5)-(6.7). In this case, one obtains the frequency-response equation

$$
\begin{gathered}
\frac{4 \Gamma_{n}^{2} \alpha_{5}^{2} B_{4}^{2} \Lambda^{6}}{\alpha_{1}^{2}}=\omega_{n}^{2} \mu_{n}^{2} a_{n}^{2} \\
{\left[\left(+\frac{\alpha_{1}+\Gamma_{n}}{\alpha_{1}}\right) \omega_{n} a_{n} \sigma-\frac{\Gamma_{n} \alpha_{5}}{\alpha_{1}}\left(\frac{1}{4} B_{1} a_{n}^{3}+B_{3} a_{n} \Lambda^{2}\right)\right]^{2}}
\end{gathered}
$$

Unlike subharmonic resonances, trivial solutions for the case of superharmonic resonance cannot exist. This type of behavior has already been seen in the case of primary resonance. 
It follows from equation (6.8) that a direct relation between the detuning and the response amplitude can be obtained as

$$
\begin{gathered}
\left(\frac{\alpha_{1}+\Gamma_{n}}{\alpha_{1}}\right) \omega_{n} \sigma=\frac{\Gamma_{n} \alpha_{5}}{\alpha_{1}}\left(\frac{1}{4} B_{1} a_{n}^{2}+B_{3} \Lambda^{2}\right) \\
\mp\left[\frac{4 \Gamma_{n}^{2} \alpha_{5}^{2} B_{4}^{2} \Lambda^{6}}{\alpha_{1}^{2} a_{n}^{2}}-\omega_{n}^{2} \mu_{n}^{2}\right]^{1 / 2}
\end{gathered}
$$

A representative frequency-response curve is shown in figure 7 . A frequencyresponse curve for the case of primary resonance is also included in this figure. The characterestics of the curves in both cases are very similar except that the curve representing the superharmonic resonance is shifted to the right for hardning springs and to the left for softning springs. This shift in frequency is due to the term $\frac{\Gamma_{n} \alpha_{5}}{\alpha_{1}} B_{3} \Lambda^{2}$ which is present in the frequency-response equation (6.9) but not in (4.45).

As discussed in the previous two chapters, Dokainish and Elmadany[5] neglected the second-order harmonic in their analysis of superharmonic resonances. To use the method of harmonic balance to determine a solution valid for general static pressure loads, one needs to assume the following solution of $(2.8)-(2.10)$ :

$$
u=A x+B_{1 / 3} \sin \frac{\lambda}{3} x \sin \frac{\Omega}{3} t+B_{1} \sin \lambda x \sin \Omega t+B_{2} \sin 2 \lambda x \sin 2 \Omega t
$$

It is clear from the development above that, to obtain a consistent solution by using the method of harmonic balance one needs to know a great deal about the solution a priori or to carry enough terms in the solution and check the order of the coeffi- 


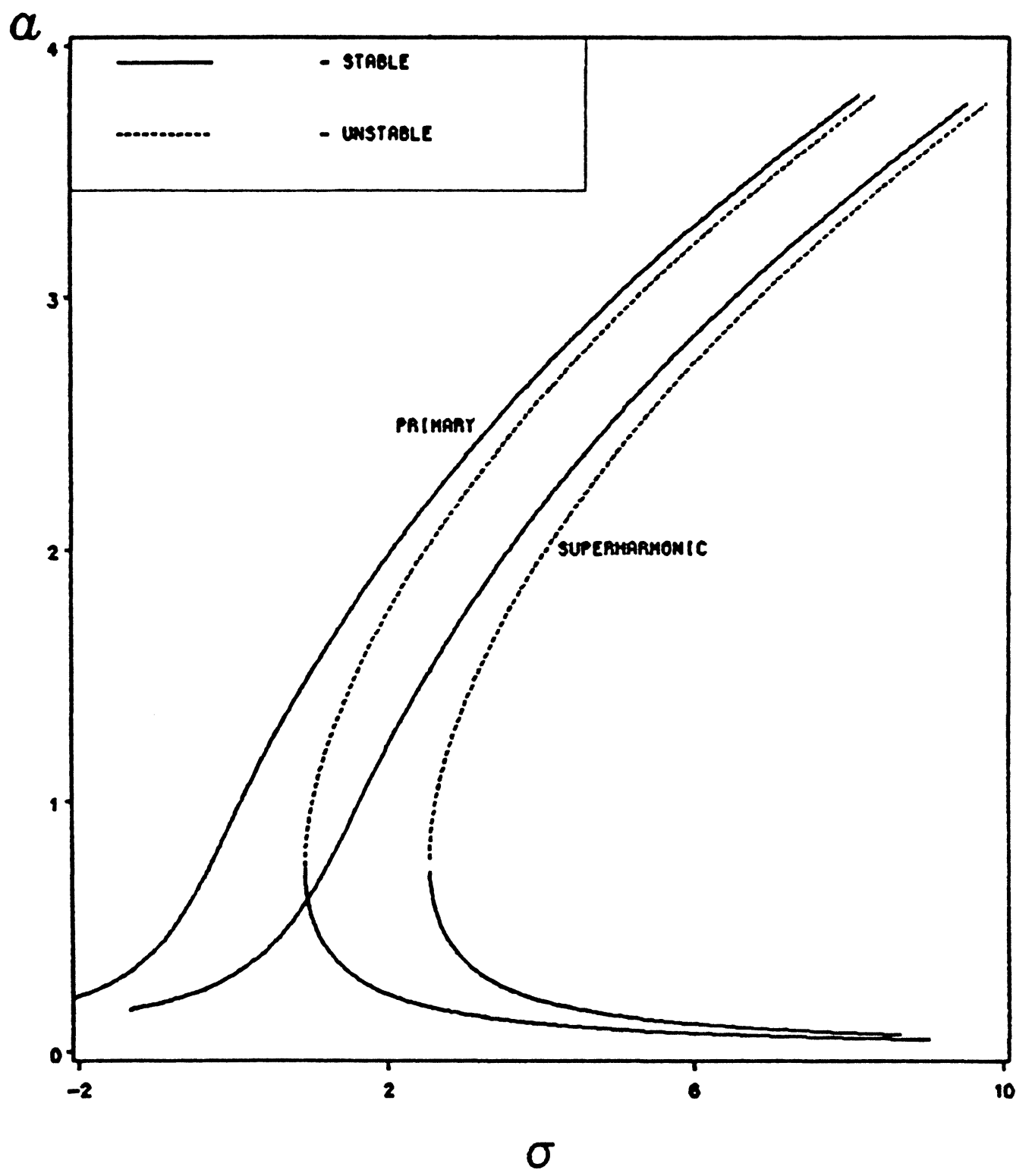

Figure 7. Comparison of frequency-response curves:

primary resonance $\left(\alpha_{1}=2, \alpha_{2}=20, \alpha_{3}=-4, f_{1}=35, \mu=.05\right)$

superharmonic resonance of order $3\left(\alpha_{1}=2, \alpha_{2}=20, \alpha_{3}=-4, f_{1}=20, \mu=.05\right)$ 
cients of all the neglected harmonics. Otherwise one might obtain an inaccurate approximation. 


\section{Case of Subharmonic Resonance of Order}

\section{One-Half}

This resonance occurs whenever the excitation frequency $\Omega$ is near twice the natural frequency of the system $\omega_{n}$. To quantitatively relate the two frequencies, one introduces the detuning parameter $\sigma$ and writes

$$
\Omega=2 \omega_{n}+\varepsilon^{2} \sigma
$$

To analyse subharmonic resonances generated by quadratic non-linearities, one needs to reorder the system of equations (3.7)-(3.9) in such a way that the excitation and the quadratic non-linearities appear at the same level in the perturbation scheme; that is,

$$
\begin{array}{cc}
w_{x x}=w_{t t}+2 \varepsilon^{2} \mu w_{t} & \\
w=0 & \text { at } x=0 \\
w_{t t}+\alpha_{1} w_{x}+\alpha_{4} w+\varepsilon \alpha_{5} w^{2}+\varepsilon^{2} \alpha_{3} w^{3}=\varepsilon f_{1} \cos \Omega t & \text { at } x=1
\end{array}
$$


Substituting equation (3.12) into (7.2)-(7.4) and equating coefficients of equal powers of $\varepsilon$ on both sides of each equation yields the following three separate problems that can be solved in succession:

Order $\varepsilon^{0}$ :

$$
\begin{array}{cl}
w_{0}=0 & \text { at } x=0 \\
D_{0} w_{0}+\alpha_{1} w_{0 x}+\alpha_{0} w_{0}=0 & \text { at } x=1
\end{array}
$$

Order $\varepsilon$ :

$$
\begin{gathered}
w_{1 x x}=D_{0}^{2} w_{1}+2 D_{0} D_{1} w_{1} \\
w_{1}=0 \quad \text { at } x=0 \\
D_{0}^{2} w_{1}+\alpha_{1} w_{1 x}+\alpha_{4} w_{1}=f_{1} \cos \Omega T_{0}-2 D_{0} D_{1} w_{0}-\alpha_{5} w_{0}^{2} \text { at } x=1
\end{gathered}
$$

Order $\varepsilon^{2}$ :

$$
\begin{array}{cc}
w_{2 x x}=D_{0}^{2} w_{2}+2 D_{0} D_{2} w_{0}+2 D_{0} D_{1} w_{1}+D_{1}^{2} w_{0}+2 \mu D_{0} w_{0} \\
w_{2}=0 & \text { at } x=0 \\
D_{0}^{2} w_{2}+\alpha_{1} w_{2 x}+\alpha_{4} w_{2} & =-2 D_{0} D_{2} w_{0}-2 D_{0} D_{1} w_{1}-D_{1}^{2} w_{0} \\
& -2 \alpha_{5} w_{0} w_{1}-\alpha_{3} w_{0}^{3} \text { at } x=1
\end{array}
$$

The solution of the zeroth-order problem is 


$$
w_{0}=A_{n}\left(T_{1}, T_{2}\right) \frac{\sin \omega_{n} x}{\sin \omega_{n}} e^{i \omega_{n} T_{0}}+c c
$$

where $\omega_{n}$ is the $n$th linear undamped natural frequency defined in (4.17).

Substituting (7.14) into (7.8)-(7.10), eliminating secular terms, and solving for $w_{1}$ yields $D_{1} A=0$ and

$$
\begin{gathered}
w_{1}=\Lambda \frac{\sin \Omega x}{\sin \Omega} e^{i \Omega T_{0}}+C_{1} A_{n}^{2} \frac{\sin 2 \omega_{n} x}{\sin 2 \omega_{n}} e^{2 i \omega_{n} T_{0}} \\
-\frac{\alpha_{5}}{\alpha_{1}+\alpha_{4}} A_{n} \bar{A}_{n} x+c c
\end{gathered}
$$

where $\Lambda$ is given by (5.10) with $\Omega \cong 2 \omega_{n}$ in this case and $C_{1}$ is defined in (5.15).

Substituting (7.14) and (7.15) into (7.11)-(7.13), recalling the fact that $D_{1} A=0$, and assuming (4.28) as a solution for $w_{2}$ yields

$$
\begin{gathered}
\varphi_{n}^{\prime \prime}+\omega_{n}^{2} \varphi_{n}=2 i \omega_{n}\left(A_{n}^{\prime}+\mu_{n} A_{n}\right) \frac{\sin \omega_{n} x}{\sin \omega_{n}} \\
\varphi_{n}=0 \quad \text { at } x=0 \\
\varphi_{n}^{\prime}+\left(\frac{\alpha_{4}-\omega_{n}^{2}}{\alpha_{1}}\right) \varphi_{n}=\frac{1}{\alpha_{1}}\left[-2 i \omega_{n} A_{n}^{\prime}-2 \alpha_{5} \bar{A}_{n} \Lambda e^{l \sigma T_{2}}\right. \\
\left.-2 \alpha_{5} B A_{n}^{2} \bar{A}_{n}\right] \quad \text { at } x=1
\end{gathered}
$$

where 


$$
B=C_{1}-\frac{2 \alpha_{5}}{\alpha_{1}+\alpha_{4}}+\frac{3 \alpha_{3}}{2 \alpha_{5}}
$$

As in Appendix A, the solvability condition for $(7.16)-(7.18)$ is

$$
2 i \omega_{n}\left(A_{n}^{\prime}+\mu_{n} A_{n}\right)=-\frac{\Gamma_{n}}{\alpha_{1}}\left[2 i \omega_{n} A_{n}^{\prime}+2 \alpha_{5} \bar{A}_{n} \Lambda e^{i \sigma T_{2}}+2 \alpha_{5} B A_{n}^{2} \bar{A}_{n}\right]
$$

where $\Gamma_{n}$ and $\mu_{n}$ are defined by (4.35) and (4.36), respectively. Substituting the polar form (4.37) into (7.20) and separating real and imaginary parts, one obtains

$$
\begin{aligned}
& \left(\frac{\alpha_{1}+\Gamma_{n}}{\alpha_{1}}\right) \omega_{n} \dot{a}_{n}=-\omega_{n} \mu_{n} a_{n}-\frac{\Gamma_{n} \alpha_{5}}{\alpha_{1}} \Lambda a_{n} \sin \gamma \\
& \omega_{n}\left(\frac{\alpha_{1}+\Gamma_{n}}{\alpha_{1}}\right) a_{n} \dot{\beta}_{n}=\frac{\Gamma_{n} \alpha_{5}}{\alpha_{1}}\left(\Lambda a_{n} \cos \gamma+\frac{1}{4} B a_{n}^{3}\right)
\end{aligned}
$$

where

$$
\gamma=\sigma T_{2}-2 \beta_{n}
$$

The steady-state motion can be determined by letting

$$
\dot{\mathrm{a}}=0, \dot{\gamma}=0
$$

Substituting (7.24) into (7.21) and (7.22) and eliminating $\gamma$ from the result yields

$$
\left[\frac{\left(\alpha_{1}+\Gamma_{n}\right)}{2 \alpha_{1}} \omega_{n} a_{n} \sigma-\frac{\Gamma_{n} \alpha_{5}}{4 \alpha_{1}} B a_{n}^{3}\right]^{2}=\left(\frac{\Gamma_{n}^{2} \alpha_{5}^{2} \Lambda^{2}}{\alpha_{1}^{2}}-\omega_{n}^{2} \mu_{n}^{2}\right) a_{n}^{2}
$$

It follows from equation (7.25) that either $a_{n}=0$ or 


$$
\frac{\Gamma_{n} \alpha_{5}}{4 \alpha_{1}} \mathrm{Ba}_{n}^{2}=\frac{\left(\alpha_{1}+\Gamma_{n}\right)}{2 \alpha_{1}} \omega_{n} \sigma \mp\left[\frac{\Gamma^{2} \alpha_{5}^{2} \Lambda^{2}}{\alpha_{1}^{2}}-\omega_{n}^{2} \mu_{n}^{2}\right]^{1 / 2}
$$

The response amplitude given by $(7.26)$ is plotted in figure 8 as a function of the detuning. Depending on the value of $\sigma$, one, two or three solutions are possible. When $\sigma<\sigma_{1}$, only the trivial solution is possible, which is stable. When $\sigma_{1}<\sigma<\sigma_{2}$, there are two possible solutions: the trivial solution, which is unstable and a nontrivial solution, which is stable. When $\sigma>\sigma_{2}$, there are three possible solutions: the trivial solution, which is stable, and two nontrivial solutions, the larger of which is stable. In the latter case the response depends on the initial conditions. It seems from figure 8 that subharmonic resonances can be excited for large values of the detuning $\sigma$, physically this is not the case. In fact, the present analysis is valid only when the excitation frequency is within a narrow interval near twice the natural frequency of the system. Also carrying out the analysis to higher order is expected to yield frequency-response curves in which the upper and lower branches merge and terminate at a finite value of $\sigma$.

Figure 9 shows a representative variation of the response amplitude with the excitation amplitude. This is the case where $(7.26)$ can have two nontrivial solutions. When $F<F_{1}$, only the trivial solution is possible, which is stable. When $F_{1}<F<$ $F_{2}$, there are three possible solutions: the trivial solution, which is stable, and two non-trivial solutions, the larger of which is stable. When $F>F_{2}$, there are two possible solutions: the trivial solution, which is unstable, and a non-trivial solution, which is stable. Thus subharmonic resonances of order one-half are not excited when $F$ $<F_{1}$, may or may not be excited depending on the initial conditions, when $F_{1}<F$ $<F_{2}$, and are always excited when $F>F_{2}$. If an experiment is carried out with a fixed $\sigma$ and slowly varying $F$ from a point below $F_{1}$, the response will be trivial until $F$ re- 


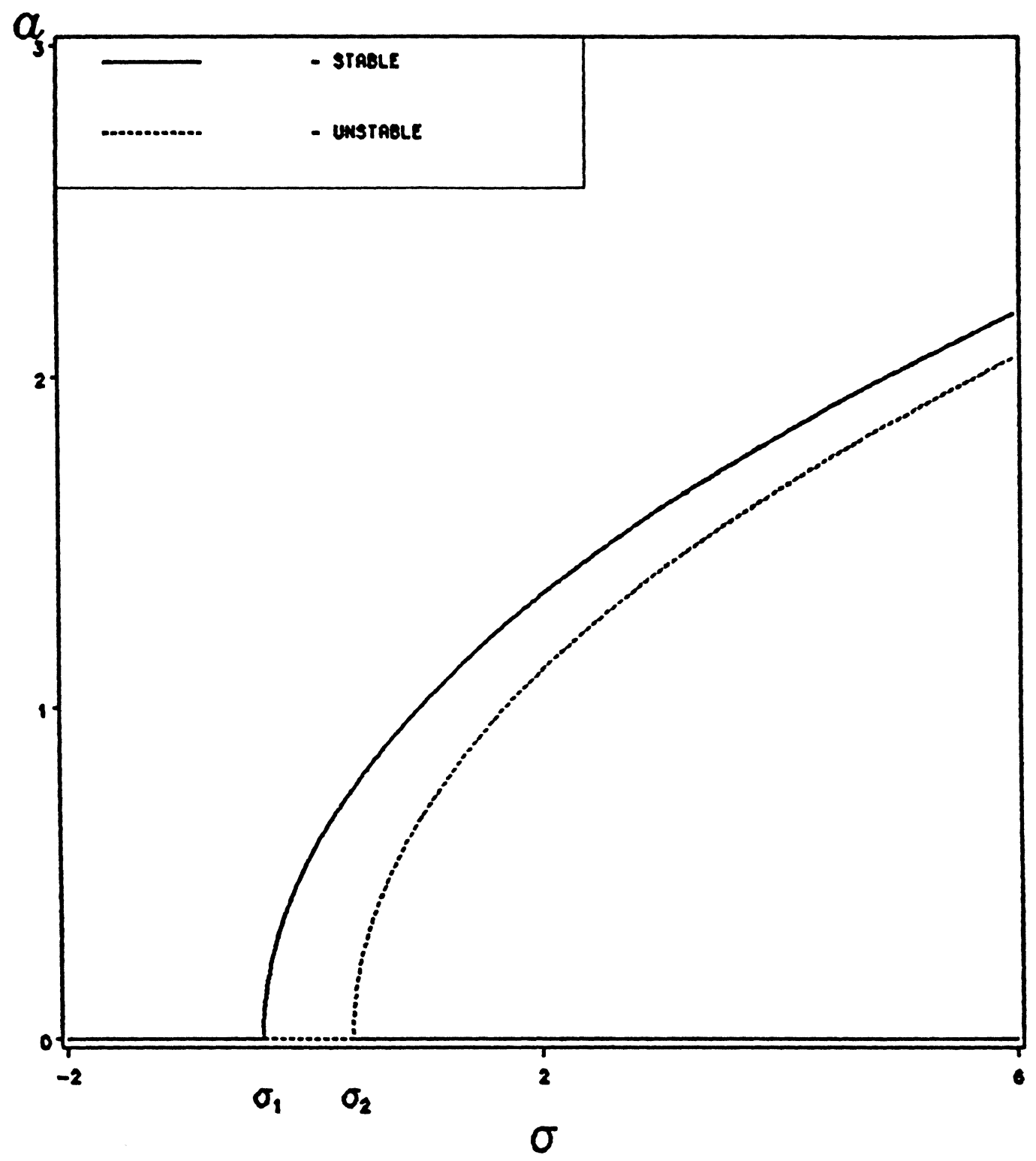

Figure 8. Frequency-response curve for the case of subharmonic resonance of order one-half $\left(\alpha_{1}=2, \alpha_{2}=20, \alpha_{3}=-6, f_{0}=20, f_{1}=20, \mu=.005\right)$ 


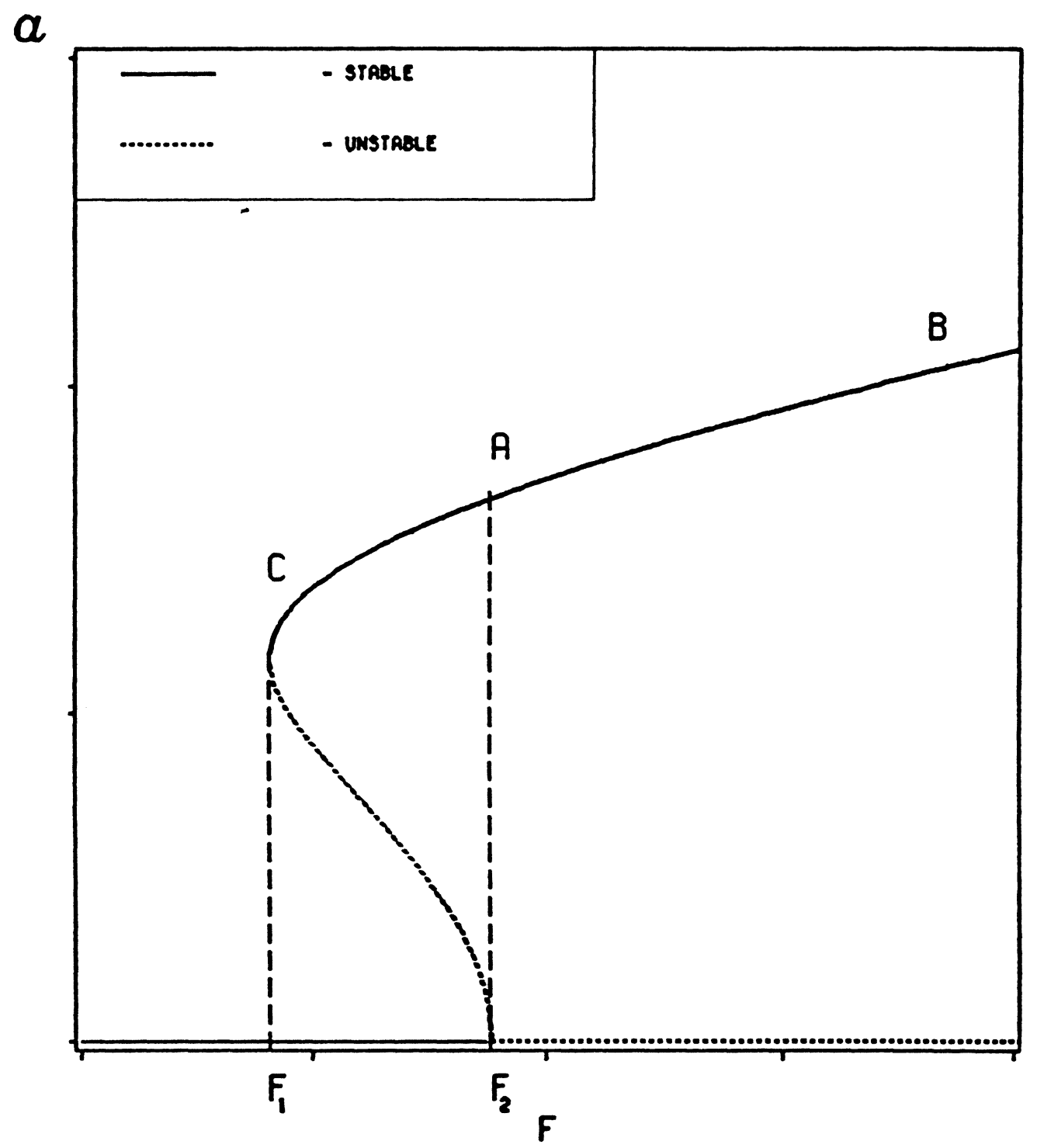

Figure 9. Response amplitude as a function of the excitation amplitude for the case of subharmonic resonance of order one-half

$\left(\alpha_{1}=2, \alpha_{2}=20, \alpha_{3}=-7, f_{0}=20, \sigma=2, \mu=.5\right)$ 


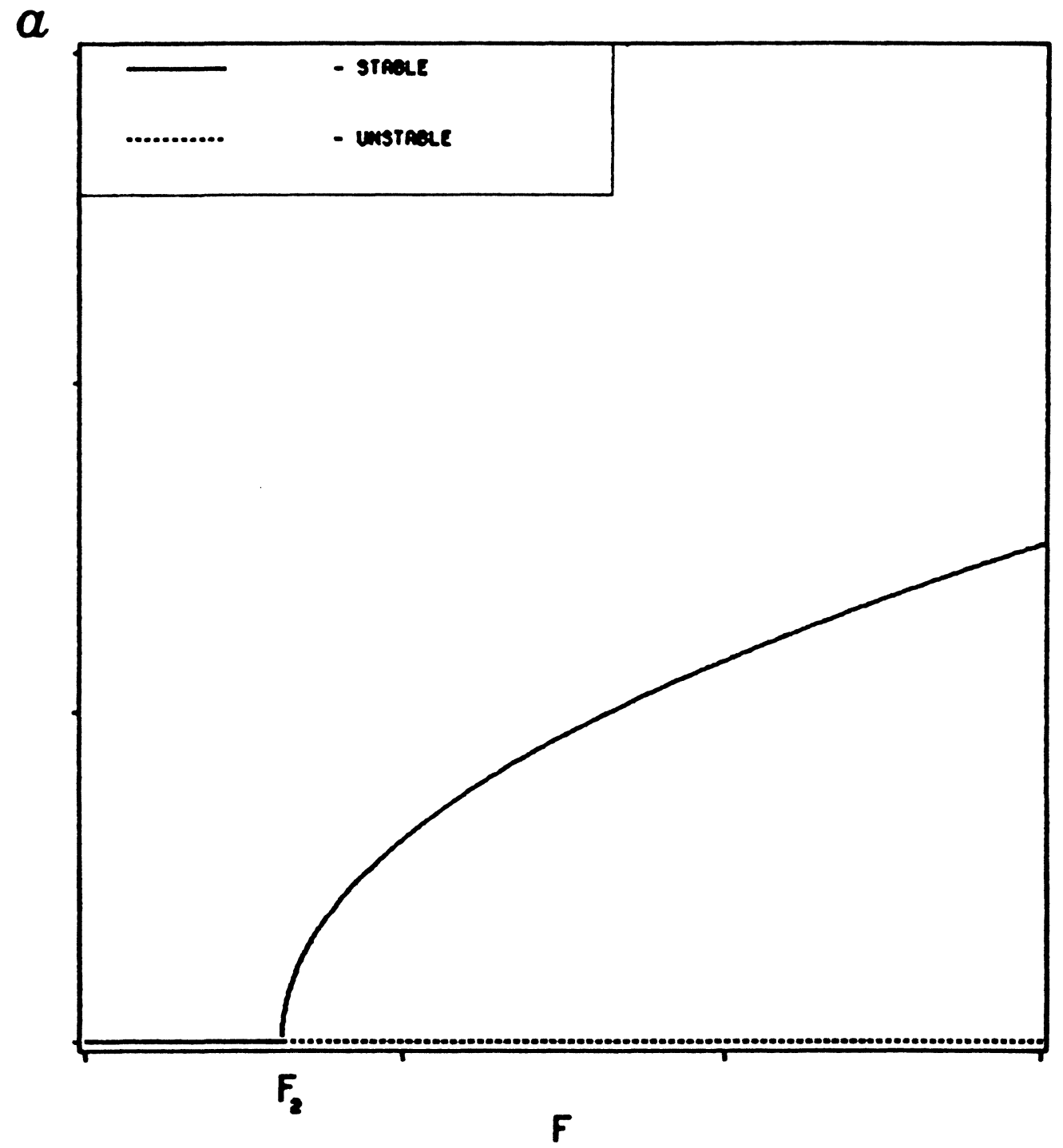

Figure 10. Response amplitude as a function of the excitation amplitude for the case of subharmonic resonance of order one-half

$\left(\alpha_{1}=2, \alpha_{2}=20, \alpha_{3}=-7, f_{0}=20, \sigma=-2, \dot{\mu}=.5\right)$ 
aches $F_{2}$. A small increase in $F$ beyond $F_{2}$ results in a sudden increase in the response amplitude. The jump occurs from position $F_{2}$ where the response is zero to position $A$ on the figure beyond which the amplitude continues to increase gradually toward point $B$ as the excitation amplitude increases. On the other hand, if one starts at point $B$. Decrasing slowly the excitation amplitude from a value of $F$ corresponding to point $B$, the response will gradually decrease until point $C$ is reached. Decreasing F further results in a downward jump of a to zero and the response becomes trivial.

Figure 10 shows another representative variation of the response amplitude with the excitation amplitude when (7.26) admits only one non-trivial solution. In this case, there is only one bifurcation value and there are no jumps in the response. 


\section{Case of Combination Resonance}

This resonance occurs whenever the excitation frequency $\Omega$ is near the sum or the difference of two natural frequncies of the system. First, we consider the case of combination resonance of the additive type. To quantitatively describe the nearness of this resonance, we introduce the detuning parameter $\sigma$ defined according to

$$
\Omega=\omega_{n}+\omega_{m}+\varepsilon^{2} \sigma
$$

The results in this case are similar to those in the previous case except that two different modes are involved instead of only one. Thus the solution of (7.5)-(7.7) is taken in the form

$$
w_{0}=A_{m}\left(T_{1}, T_{2}\right) \frac{\sin \omega_{m} x}{\sin \omega_{m}} e^{i \omega_{m} T_{0}}+A_{n}\left(T_{1}, T_{2}\right) \frac{\sin \omega_{n} x}{\sin \omega_{n}} e^{i \omega_{n} T_{0}}+c c
$$

Substituting (8.2) into (7.8)-(7.10), eliminating the terms that produce secular terms, and solving for $w_{1}$, we have 


$$
\begin{aligned}
w_{1}= & \Lambda \frac{\sin \Omega x}{\sin \Omega} e^{i \Omega T_{0}}+C_{1} A_{n}^{2} \frac{\sin 2 \omega_{n} x}{\sin 2 \omega_{n}} e^{2 i \omega_{n} T_{0}} \\
- & \frac{\alpha_{5}}{\alpha_{1}+\alpha_{4}} A_{n} \bar{A}_{n} x+C_{1} A_{m}^{2} \frac{\sin 2 \omega_{m} x}{\sin 2 \omega_{m}} e^{2 i \omega_{m} T_{0}} \\
- & \frac{\alpha_{5}}{\alpha_{1}+\alpha_{4}} A_{m} \bar{A}_{m} x+C_{3} A_{n} A_{m} \frac{\sin \left(\omega_{n}+\omega_{m}\right) x}{\sin \left(\omega_{n}+\omega_{m}\right)} e^{1\left(\omega_{n}+\omega_{m}\right) T_{0}} \\
& +C_{4} A_{n} \bar{A}_{m} \frac{\sin \left(\omega_{n}-\omega_{m}\right) x}{\sin \left(\omega_{n}-\omega_{m}\right)} e^{i\left(\omega_{n}-\omega_{m}\right) T_{0}}+c c
\end{aligned}
$$

Substituting (8.2) and (8.3) into (7.11)-(7.13), recalling the fact that $D_{1} A=0$, and assuming the form (4.28) for $w_{2}$, we obtain

$$
\begin{gathered}
\varphi^{\prime \prime}{ }_{n}+\omega_{n}^{2} \varphi_{n}=2 i \omega_{n}\left(A_{n}^{\prime}+\mu_{n} A_{n}\right) \frac{\sin \omega_{n} x}{\sin \omega_{n}} \\
\varphi_{n}=0 \quad \text { at } \quad x=0 \\
\varphi_{n}^{\prime}+\left(\frac{\alpha_{4}-\omega_{n}^{2}}{\alpha_{1}}\right) \varphi_{n}=\frac{1}{\alpha_{1}}\left[-2 i \omega_{n} A_{n}^{\prime}\right. \\
\left.-2 \alpha_{5}\left(B_{1} A_{n}^{2} \bar{A}_{n}+B_{2} A_{n} A_{m} \bar{A}_{m}+\bar{A}_{m} \Lambda e^{l \sigma T_{2}}\right)\right] \text { at } x=1
\end{gathered}
$$

and

$$
\begin{gathered}
\varphi_{m}^{\prime \prime}+\omega_{m}^{2} \varphi_{m}=2 i \omega_{m}\left(A_{m}^{\prime}+\mu_{m} A_{m}\right) \frac{\sin \omega_{m} x}{\sin \omega_{m}} \\
\varphi_{m}=0 \\
\varphi_{m}^{\prime}+\left(\frac{\alpha_{4}-\omega_{m}^{2}}{\alpha_{1}}\right) \varphi_{m}=\frac{1}{\alpha_{1}}\left[-2 i \omega_{m} A_{m}^{\prime}\right. \\
\left.-2 \alpha_{5}\left(B_{3} A_{m}^{2} \bar{A}_{m}+B_{2} A_{m} A_{n} \bar{A}_{n}+\bar{A}_{n} \Lambda e^{i \sigma T_{2}}\right)\right] \text { at } x=1
\end{gathered}
$$


where $\Lambda$ is defined in (5.7),

$$
\begin{gathered}
\mathrm{B}_{1}=\mathrm{C}_{1}-\frac{2 \alpha_{5}}{\alpha_{1}+\alpha_{4}}+\frac{3 \alpha_{3}}{2 \alpha_{5}} \\
\mathrm{~B}_{2}=\mathrm{C}_{3}+\mathrm{C}_{4}-\frac{2 \alpha_{5}}{\alpha_{1}+\alpha_{4}}+\frac{3 \alpha_{3}}{2 \alpha_{5}} \\
\mathrm{~B}_{3}=\mathrm{C}_{2}-\frac{2 \alpha_{5}}{\alpha_{1}+\alpha_{4}}+\frac{3 \alpha_{3}}{2 \alpha_{5}}
\end{gathered}
$$

Here $C_{1}$ is given by $(5.15), C_{2}$ can also be given by $(5.16)$ by interchanging $\omega_{n}$ with $\omega_{m}$, and $C_{3}$ and $C_{4}$ can be found from $(5.17)$ and $(5.18)$, respectively, by simply interchanging $\Omega$ with $\omega_{m}$. The solvability conditions for (8.4)-(8.6) and (8.7)-(8.9) can be determined as

$$
\begin{gathered}
\left(\frac{\alpha_{1}+\Gamma_{n}}{\alpha_{1}}\right) \omega_{n} \dot{a}_{n}=-\omega_{n} \mu_{n} a_{n}-\frac{\Gamma_{n} \alpha_{5}}{\alpha_{1}} a_{m} \Lambda \sin \gamma \\
\omega_{n}\left(\frac{\alpha_{1}+\Gamma_{n}}{\alpha_{1}}\right) a_{n} \dot{\beta}_{n}=\frac{\Gamma_{n} \alpha_{5}}{\alpha_{1}}\left[\frac{1}{4} B_{1} a_{n}^{3}+\frac{1}{4} B_{2} a_{n} a_{m}^{2}+a_{m} \Lambda \cos \gamma\right] \\
\left(\frac{\alpha_{1}+\Gamma_{m}}{\alpha_{1}}\right) \omega_{m} \dot{a}_{m}=-\omega_{m} \mu_{m} a_{m}-\frac{\Gamma_{m} \alpha_{5}}{\alpha_{1}} a_{n} \Lambda \sin \gamma \\
\omega_{m}\left(\frac{\alpha_{1}+\Gamma_{m}}{\alpha_{1}}\right) a_{m} \dot{\beta}_{m}=\frac{\Gamma_{m} \alpha_{5}}{\alpha_{1}}\left[\frac{1}{4} B_{3} a_{m}^{3}+\frac{1}{4} B_{2} a_{m} a_{n}^{2}+a_{n} \Lambda \cos \gamma\right]
\end{gathered}
$$

where

$$
\gamma=\sigma T_{2}-\beta_{n}-\beta_{m}
$$


For steady-state motion, $\dot{a}_{n}=\dot{a}_{m}=0$ and $\dot{\gamma}=0$. It follows from equations (8.13) and (8.15) that

$$
a_{m}=a_{n}\left[\frac{\omega_{n} \mu_{n} \Gamma_{m}}{\omega_{m} \mu_{m} \Gamma_{n}}\right]^{1 / 2}
$$

Then, it follows from equations $(8.14),(8.16)$ and $(8.18)$ that the steady-state solution is either described by the trivial solution $a_{n}=a_{m}=0$ or

$$
a_{n}=\left[\frac{\Gamma_{n}}{\alpha_{1} S_{1}} \sigma-\frac{S_{2}}{S_{1}}\left(\frac{\Gamma_{n}^{2} \alpha_{5}^{2}}{\alpha_{1}^{2}} \Lambda^{2} S_{3}-\omega_{n}^{2} \mu_{n}^{2}\right)^{1 / 2}\right]^{1 / 2}
$$

where

$$
\begin{gathered}
S_{1}=\frac{\alpha_{5}}{4 \alpha_{1}}\left[\frac{\Gamma_{n}}{\omega_{n}\left(\alpha_{1}+\Gamma_{n}\right)}\left(B_{1} \Gamma_{n}+B_{2} \frac{\mu_{n} \omega_{n}}{\mu_{m} \omega_{m}} \Gamma_{m}\right)\right. \\
\left.+\frac{\Gamma_{m}}{\omega_{m}\left(\alpha_{1}+\Gamma_{m}\right)}\left(B_{2} \Gamma_{n}+B_{3} \frac{\mu_{n} \omega_{n}}{\mu_{m} \omega_{m}} \Gamma_{m}\right)\right] \\
S_{2}=\frac{\Gamma_{n}}{\omega_{n}}\left(\frac{1}{\alpha_{1}+\Gamma_{n}}+\frac{\mu_{m}}{\mu_{n}} \frac{1}{\alpha_{1}+\Gamma_{m}}\right) \\
S_{3}=\frac{\omega_{n} \mu_{n} \Gamma_{m}}{\omega_{m} \mu_{m} \Gamma_{n}}
\end{gathered}
$$

The results for the case $\Omega=\omega_{n}-\omega_{m}$ can be found by simply changing the sign of $\omega_{m}$. However, it follows from (8.18) that combination resonances of the difference type cannot be excited. 


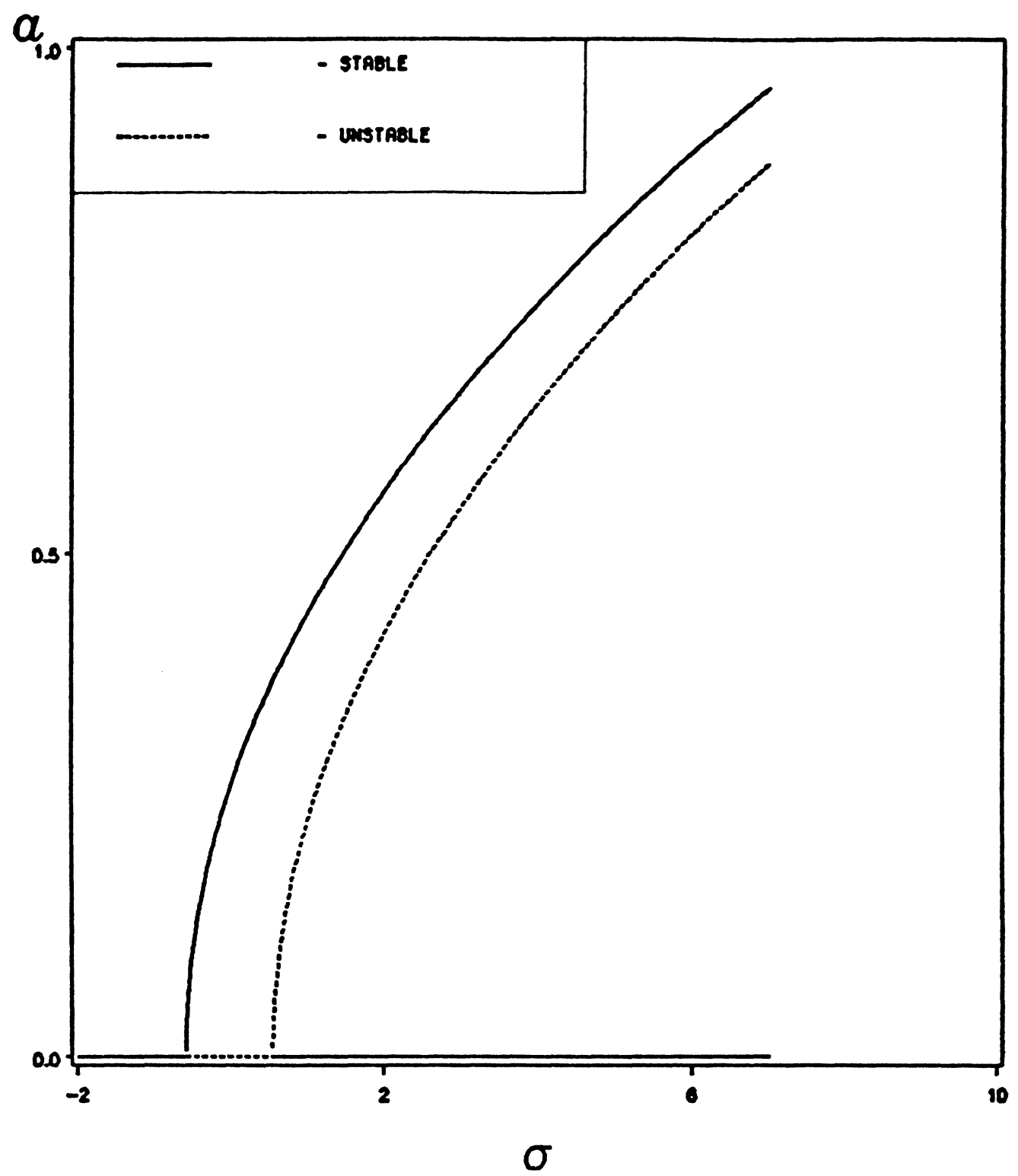

Figure 11. Frequency-response curve for the case of combination resonance of the additive type $\left(\alpha_{1}=2, \alpha_{2}=20, \alpha_{3}=-6, f_{0}=20, f_{1}=20, \mu=.001\right)$ 
Figure 11 shows a representative variation of the response amplitude with the detuning for the case of combination resonance of the additive type. We note that the frequency-response equation (8.17) has the same form as the frequency-response equation (7.26) obtained for the case of subharmonic resonances of order one-half. 


\section{Case of Superharmonic resonance of order 2}

The case of superharmonic resonance of order 2 occurs whenever the excitation frequency is near one-half one of the natural frequencies of the system. To treat this type of resonance, one needs to choose $\lambda$ and $\mu_{1}$ in (3.7)-(3.9) so that

$$
\begin{array}{cc}
w_{x x}=w_{t t}+2 \varepsilon^{2} \mu w_{t} & \\
w=0 & \text { at } x=0 \\
w_{t t}+\alpha_{1} w_{x}+\alpha_{4} w+\varepsilon \alpha_{5} w^{2}+\varepsilon^{2} \alpha_{3} w^{3}=\varepsilon^{1 / 2} f_{1} \cos \Omega t & \text { at } x=1
\end{array}
$$

If one neglects the damping and the non-linear terms from (9.1)-(9.3) and solves the resulting problem, one obtains

$$
w=\varepsilon^{1 / 2} w_{L}=\varepsilon^{1 / 2} \Lambda \frac{\sin \Omega x}{\sin \Omega} e^{i \Omega t}+c c
$$

where $\Lambda$ is given by (5.10).

Now let 


$$
w=\varepsilon^{1 / 2} w_{L}+v
$$

in (3.7)-(3.9) and obtain

$$
\begin{gathered}
v_{x x}=v_{t t}+2 \varepsilon^{2} \mu v_{t}+\cdots \\
v=0 \\
v_{t t}+\alpha_{1} v_{x}+\alpha_{4} v+\varepsilon \alpha_{5} v^{2}+\varepsilon^{2} \alpha_{3} v^{3}=-2 \varepsilon^{3 / 2} v \Lambda e^{i \Omega t}-\varepsilon^{2} \alpha_{5} \Lambda^{2} e^{2 i \Omega t}+\cdots \text { at } x=1(9.8)
\end{gathered}
$$

The problem has been transformed into that of determining the solution of (9.6)-(9.8) for the case of primary resonance (i.e., $2 \Omega \cong \omega_{n}$ ).

The nearness of this type of resonance can be expressed quantitatively by introducing the detuning parameter $\sigma$ defined according to

$$
2 \Omega=\omega_{n}+\varepsilon^{2} \sigma
$$

Following steps similar to those in section 4 and considering only the nth mode, we find the following solvability condition:

$$
2 i \omega_{n}\left(A_{n}^{\prime}+\mu_{n} A_{n}\right)=-\frac{\Gamma_{n}}{\alpha_{1}}\left[2 i \omega_{n} A_{n}^{\prime}+\alpha_{5}\left(2 B A_{n}^{2} \bar{A}_{n}+\Lambda^{2} e^{l \sigma T_{2}}\right)\right]
$$

where $\Gamma_{n}$ and $\mu_{n}$ are defined by (4.35) and (4.36), respectively, and $B$ is defined by (4.32).

Substituting the polar form (4.37) into (9.10) and separating real and imaginary parts, one obtains 


$$
\begin{aligned}
& \left(\frac{\alpha_{1}+\Gamma_{n}}{\alpha_{1}}\right) \omega_{n} \dot{a}_{n}=-\omega_{n} \mu_{n} a_{n}-\frac{\Gamma_{n} \alpha_{5}}{\alpha_{1}} \Lambda^{2} \sin \gamma \\
& \omega_{n}\left(\frac{\alpha_{1}+\Gamma_{n}}{\alpha_{1}}\right) a_{n} \dot{\beta}_{n}=\frac{\Gamma_{n} \alpha_{5}}{\alpha_{1}}\left(\frac{1}{4} B a_{n}^{3}+\Lambda^{2} \cos \gamma\right)
\end{aligned}
$$

where

$$
\gamma=\sigma T_{2}-\beta_{n}
$$

The steady-state motion can be determined by letting $\mathrm{a}=0$ and $\dot{\gamma}=0$ in (9.11)-(9.13). It follows from (9.13) that, $\dot{\beta}_{n}=\sigma$. Then, (9.11) and (9.12) can be combined to produce the frequency-response equation

$$
\left(\frac{\alpha_{1}+\Gamma_{n}}{\alpha_{1}}\right) \omega_{n} \sigma=\frac{\Gamma_{n} \alpha_{5}}{4 \alpha_{1}} B a_{n}^{2} \mp\left[\frac{\Gamma_{n}^{2} \alpha_{5}^{2} \Lambda^{4}}{\alpha_{1}^{2} a_{n}^{2}}-\omega_{n}^{2} \mu_{n}^{2}\right]^{1 / 2}
$$

This equation is similar to (4.45) obtained for the case of primary resonances if one identifies $\Lambda^{2}$ with $\frac{1}{2} f_{1}$. 


\section{Summary and Conclusions}

The method of multiple scales was used to analyze the non-linear response of a relief valve under static and dynamic loading. First-order differential equations were derived for the evolution of the amplitudes and the phases for the following resonances: primary resonance; subharmonic resonance of order one-third; superharmonic resonance of order three; subharmonic resonance of order one-half; superharmonic resonance of order two; and combination resonances of both the additive and the difference types. The evolution equations were used to determine the steady-state motion and the results were then used to obtain representative frequency-response curves for each case of resonance. The stability of the steady-state solutions was investigated. It was observed both stable and unstable solutions may exist and may cause unsatisfactory performance of the relief valve. It was also observed that, unlike linear systems, multi-valued responses are possible for nonlinear systems. Thus, the initial conditions determine the response. The multi-valuedness of the solution is the cause of the jump phenomenon observed in most cases of resonances.

In the case of subharmonic resonance of order one-third, it was learned that increasing the excitation amplitude may result in a decrease rather than an increase in 
the response amplitude. It was also observed that this resonance is only excited within a certain interval of excitation amplitude and is not excited outside this interval. In the case of subharmonic resonance of order one-half, it was observed that this resonance is not excited for excitation amplitudes below a certain value, but start to exist as soon as that value is exceeded. Thereafter, the response continues to grow gradually as the excitation amplitude is increased.

Finally, the results obtained by the method of multiple scales were compared with those obtained by Dokainish and Elmadany using the method of harmonic balance. The latter were found to have limited validity. This is the result of their assumed solution. We note that to obtain a consistent approximation using the method of harmonic balance, one needs to know the form of the solution a priori. Therefore the method of multiple scales, which does not require any prior knowledge about the solution and still gives consistent approximation, is preferred over the method of harmonic balance. 


\section{References}

1. Ablowitz, M. J., Askar, A., Cakmak, A. S. and Engin, H., " Resonant non-linear vibrations in continuous systems-I : Undamped case, "International Journal of Non-linear Mechanics 14, 1977, pp 223-233.

2. Collins, W. D., " Forced oscillations of systems governed by one-dimensional non-linear wave equations," Quarterly Journal of Mechanics and Applied Mathematics 1971, vol. 24, pp 129-153.

3. Dokainish, M. A. and Elmadany, M. M., " On the nonlinear response of a fluid valve," Transactions of ASME, Journal of Applied Mechanics vol. 100, pp 675-680.

4. Hayashi, C., Nonlinear Oscillations in Physical Systems, McGraw-Hill, New York, 1964.

5. Hayashi, S., Matsui, T., Imaik, K., "Stability and self-sustained oscillations in nozzle-flapper valve with pipe line, "Bulletin of JSME, Vol. 23, No. 179, May 1980, pp 759-765.

6. Johnson, B. L. and Wandling, D. E., "Actual popping pressure of a relief valve with a real helical spring under dynamic load," Journal of Engineering for Industry, Transactions of ASME, Nov. 1969, pp 1142-46. 
7. Johnson, B. L. and Stewart, E. E., " Transfer functions for helical springs," Journal of Engineering for Industry, Transactions of ASME, Nov. 1969, pp 1011-1016.

8. Meirovitch, L., Computational Methods in Structural Dynamics, Sijthoff \& Noordohoff, Rockville, MD, 1980.

9. Nakashima, T., Shiramoto, K., Kondo T. and Sasaguchi, K., "A study on unstable phenomenon and resonance of a pressure regulating valve, "Bulletin of JSME, Vol. 29, No. 248, Feb. 1986, pp 459-465.

10. Nayfeh, A. H., Introduction to Perturbation Techniques, Wiley-Interscience, New York, 1981.

11. Nayfeh, A H. and Mook, D. T., Nonlinear Oscillations, Wiley-Interscience, New York, 1979.

12. Nayfeh, A. H. and Asfar, K. R., " Response of a bar constrained by a non-linear spring to a harmonic excitation," Journal of Sound and Vibrations 1986 105(1),1-15.

13. Nayfeh, A. H., " The response of single-degree of freedom systems with quadratic and cubic non-linearities to a subharmonic excitation," Journal of Sound and Vibrations 1983 , Vol. 89 , pp 457-470.

14. Paslay, P. R. and Gurtin, M. E., " The vibration response of linear undamped system resting on a non-linear spring," Journal of Applied Mechanics, Transactions of ASME, June 1980, pp 272-274.

15. Stoker, J. J., Nonlinear Vibrations in Mechanical and Electrical Systems, Interscience, New York, 1950.

16. Wahl, A. M., Mechanical Springs, McGraw-Hill, New York, N. Y., 2nd ed., 1963.

17. Watanabe, T., "Forced vibration of continuous system with nonlinear boundary conditions, " Transaction of ASME , Journal of Mechanical Design 100, 1978, pp 487-491. 


\section{Appendix A. Solvability Conditions}

Consider the problem

$$
\begin{array}{ll}
\varphi^{\prime \prime}+\omega^{2} \varphi=f(x) & \text { at } \quad x=0 \\
\varphi=0 \quad \text { at } \quad x=1 \\
\varphi^{\prime}+\alpha \varphi=c \quad \text { }
\end{array}
$$

where the homogeneous problem has a non-trivial solution. One multiplies (A1) by the adjoint function $\psi(x)$, integrates the result by parts from $x=0$ to $x=1$ to transfer the derivatives from $\varphi$ to $\psi$, and obtains

$$
\left[\varphi^{\prime} \psi-\varphi \psi^{\prime}\right]_{0}^{1}+\int_{0}^{1}\left(\psi^{\prime \prime}+\omega^{2} \psi\right) \varphi d x=\int_{0}^{1} \psi f d x
$$

The equation governing the adjoint function is obtained by setting the coefficient of $\varphi$ in the integrand in (A4) equal to zero; that is

$$
\psi^{\prime \prime}+\omega^{2} \psi=0
$$


To determine the adjoint boundary conditions, one considers the homogeneous problem. Thus, using (A2) and (A3) in (A4) with $f$ and $c$ being zero yields

$$
\left.\varphi\left(\psi^{\prime}+\alpha \psi\right)\right|_{x=1}+\varphi^{\prime}(0) \psi(0)=0
$$

Setting each of the coefficients of $\varphi(1)$ and $\varphi^{\prime}(0)$ equal to zero yields the adjoint boundary conditions

$$
\psi(0)=0, \quad \psi^{\prime}(1)+\alpha \psi(1)=0
$$

Having defined the adjoint, one can return to the inhomogeneous problem. Using (A2), (A3), (A5) and (A7) in (A4) yields the solvability condition

$$
c \psi(1)=\int_{0}^{1} \psi f d x
$$

where $\psi$ is any solution of (A5) and (A7), that is, $\psi=\sin \omega_{m} x$, where $\omega_{m}$ is given by (4.17). 


\section{Appendix B. Method of harmonic balance}

Dokainish and Elmadany [5] used the method of harmonic balance to study the harmonic oscillations of the undamped relief valve represented by (2.8)-(2.10). In their analysis they considered only the first harmonic and assumed as a solution the following:

$$
u=A x+B \sin \lambda x \sin \Omega t
$$

However, the results of the method of multiple scales (chapter 4) show that higher harmonics do interact with the first harmonic and must be included in order to obtain a consistent approximation. Consequently, the general form of the solution of (2.8)-(2.10) has the form

$$
u=A x+B_{1} \frac{\sin \lambda x}{\sin \lambda L} \cos (\Omega t-\gamma)+B_{2} \frac{\sin 2 \lambda x}{\sin 2 \lambda L} \cos (2 \Omega t-2 \gamma)
$$

where $\lambda=\frac{\Omega}{L} \sqrt{\frac{M}{K}}$.

Equation (B2) satisfies the equation of motion (2.8) as well as the left boundary condition (2.9). Substituting (B2) into the second boundary condition (2.10) yields 


$$
\begin{aligned}
& -m \Omega^{2} B_{1} \cos \varphi-4 m \Omega^{2} \cos 2 \varphi \\
& +K L\left[A+\lambda B_{1} \frac{\cos \lambda L}{\sin \lambda L} \cos \varphi+2 \lambda B_{2} \frac{\cos 2 \lambda L}{\sin 2 \lambda L} \cos 2 \varphi\right] \\
& +\alpha\left[A L+B_{1} \cos \varphi+B_{2} \cos 2 \varphi\right]+ \\
& +\beta\left[A L+B_{1} \cos \varphi+B_{2} \cos 2 \varphi\right]^{3}=\Phi_{0}+\Phi_{1} \cos \Omega t
\end{aligned}
$$

where

$$
\varphi=\Omega t-\gamma
$$

Expanding the cubic term in (B3), using trigonometric identities, and equating the coefficients of $\sin \varphi, \cos \varphi$ and $\cos 2 \varphi$ on each side, we obtain

$$
\begin{gathered}
{\left[K L+\alpha L+\frac{3}{2} \beta L B_{1}^{2}+\frac{3}{2} \beta L B_{2}^{2}\right] A} \\
+\beta A^{3} L^{3}+\frac{3}{4} \beta B_{1}^{2} B_{2}=\Phi_{0} \\
\Phi_{1} \sin \beta=0 \\
{\left[-m \Omega^{2}+\lambda K L \frac{\cos \lambda L}{\sin \lambda L}+\alpha+3 \beta A^{2} L^{2}\right.} \\
\left.+\frac{3}{2} \beta B_{2}^{2}+3 \beta A L B_{2}\right] B_{1}+\frac{3}{4} \beta B_{1}^{3}=\Phi_{1} \cos \beta \\
{\left[-4 m \Omega^{2}+2 \lambda K L \frac{\cos 2 \lambda L}{\sin 2 \lambda L}+\alpha+3 \beta A^{2} L^{2}\right] B_{2}+\frac{3}{2} \beta B_{1}^{2} B_{2}} \\
+\frac{3}{2} \beta A L B_{1}^{2}+\frac{3}{4} \beta B_{2}^{3}=0
\end{gathered}
$$


The form of the solution shows that $B_{2}<B_{1}$ and $A=A_{0}+B_{0}$, where $B_{0}<<B_{1}$. Hence, (B5) reduces to

$$
(K L+\alpha L) A_{0}+\beta A_{0}^{3} L^{3}=\Phi_{0}
$$

This is the same as (3.2) where $A$ is identified with b. Substituting (B9) into (B5) yields

$$
\left(K L+\alpha L+3 \beta A_{0}^{2} L^{3}\right) B_{0}+\frac{3}{2} \beta A_{0} L B_{1}^{2}=0
$$

or

$$
B_{0}=-\frac{3}{2} \frac{\beta A_{0} L B_{1}^{2}}{K L+\alpha L+3 \beta A_{0}^{2} L^{3}}
$$

Equation (B11) can be identified with the term $C_{1} A_{n} \bar{A}_{n}$ found in (4.22). Equation (B6) results in $\beta=n \pi$ where $n$ is an integer. Solving (B8) for $B_{2}$ gives

$$
B_{2}=-\frac{3}{2} \frac{\beta A_{0} L B_{1}^{2}}{\alpha-4 m \Omega^{2}+2 \lambda K L \frac{\cos 2 \lambda L}{\sin 2 \lambda L}+3 \beta A_{0}^{2} L^{2}}
$$

Equations (B11) and (B12) show that $B_{0}$ and $B_{2}$ are $O\left(B_{1}^{2}\right)$. Keeping terms up to $O\left(B_{1}^{3}\right)$ in (B7), one obtains

$$
\begin{aligned}
& {\left[-m \Omega^{2}+\lambda K L \frac{\cos \lambda L}{\sin \lambda L}+\alpha+3 \beta A_{0}^{2} L^{2}\right] B_{1}} \\
& +\left.6 \beta A_{0}\right|^{2} B_{0} B_{1}+3 \beta A_{0} L B_{2} B_{1}+\frac{3}{4} \beta B_{1}^{3}=\Phi_{1}
\end{aligned}
$$




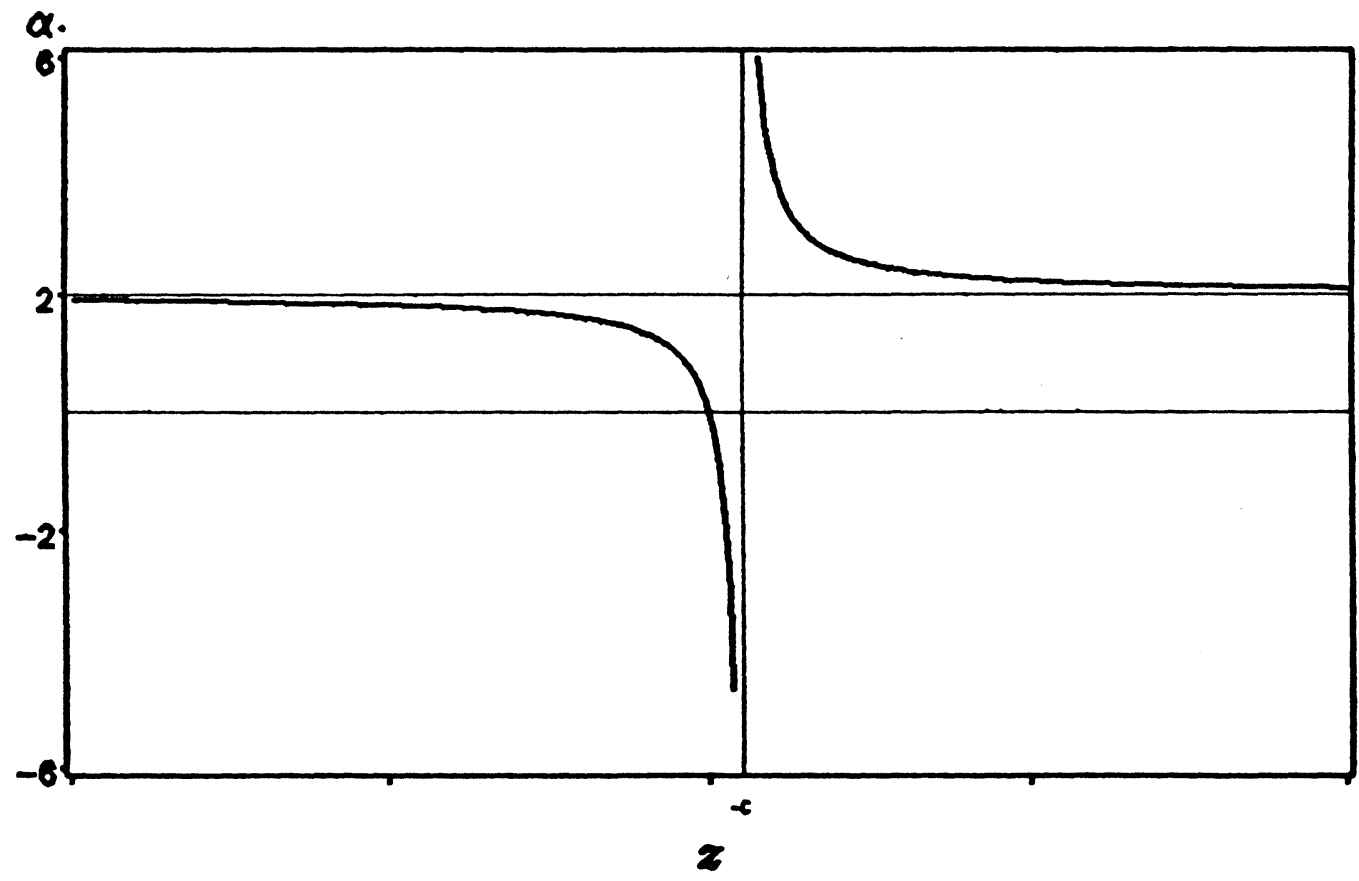

Flgure 12. Effectlve spring constant due to second harmonlc. 
Equation (B13) is the frequency-response equation and is similar to (4.45) obtained using the method of multiple scales except that the latter takes into consideration damping in the system. It includes the terms proportional to $B_{0} B_{1}$ and $B_{2} B_{1}$ which are $O\left(B_{1}^{3}\right)$ but were absent in the form assumed by Dokainish-Elmadany[5]. The term proportional to $B_{0} B_{1}$ is burried in the constant $A$ in (B1). However, the term proportional to $B_{2} B_{1}$ is totally absent from their analysis. Considering the last two terms in (B13) and substituting for $B_{2}$ from (B12), one obtains the effective non-linear spring constant

$$
\beta_{e}=\frac{3}{4} \beta\left[1-\frac{6 \beta A_{0}^{2} L^{2}}{c+3 \beta A_{0}^{2} L^{2}}\right]
$$

where

$$
c=\alpha-4 m \Omega^{2}+2 \lambda K L \frac{\cos 2 \lambda L}{\sin 2 \lambda L}
$$

Letting

$$
z=3 \beta A_{0}^{2} L^{2}
$$

and denoting by $\alpha_{0}$ the contribution of the second harmonic to the spring constant, we have

$$
\alpha_{e}=\frac{2 z}{z+c}
$$

A representative variation of $\alpha_{0}$ with $z$, for a fixed $c$ shown in figure 12 , which shows that for large values of $z, \alpha_{0}$ approaches a value of 2 . When $z$ is small, $\alpha_{0}$ is also small 
and can be neglected. When $z$ is approximatly equal to $-c, \alpha_{0}=\infty$ and the present analysis breaks down. The case $z=-c$ corresponds to the case of two-to-one internal resonance, which was excluded in the analysis. 
The vita has been removed
from the scanned document 